\title{
TOUT TON FLOTTANT BAS AUTORISE-T-IL LA PROPOGATION DU TON HAUT PRÉCÉDENT EN DAGARA-WULE?
}

\author{
Penou-Achille Somé \\ The University of Lethbridge
}

\begin{abstract}
This study addresses the syntactic implications of low floating tones in Wule, one of the three varieties of Dagara, a language of the Voltaic Group spoken in the northern part of Ghana and the southern part of Burkina Faso. The data show that if "peripheral" low floating tones allow a high preceding tone to spread, it is the other way around for "internal" low floating tones. In fact, the latter type of tones, like opaque consonants, always prevents a high preceding tone from spreading. This result leads to a question concerning the relationship between internal low floating tones and opaque consonants. After debating two different assumptions, it has been retained that, despite the similar syntactic implications, internal low floating tones and opaque consonants are two different linguistic entities which do not have, as such, any historical link.
\end{abstract}

\section{Introduction}

Nous voulons dans cet article aborder le problème du blocage du ton haut précédent par des tons flottants bas, et discuter du lien éventuel entre ces tons et les consonnes opaques qui, elles aussi, entraînent les mêmes effets tonals dans la combinatoire. Pour cela, il faut d'abord rappeler ceci: dans P. A. Somé [1995], nous avons identifié quatre schèmes tonals- $[\mathrm{BH}],[\mathrm{BB}],[\mathrm{HD}]$ (haut-Downstep) et $[\mathrm{HH}] —$ en dagara, une langue voltaïque à trois variétés (wule, birfuor, lobr) parlées à partie du nord du Ghana jusqu'au sud du Burkina Faso [cf. aussi D. J. Somé 1975, 1983]. L'analyse a montré que la nature phonologique de ces schèmes ne coincidait pas avec la forme phonétique, et que chacun d'entre eux était marqué par un ton flottant, haut ou bas, ou par deux tons flottants dans le cas unique de $\mathrm{HD}$. C'est ainsi que le schème $[\mathrm{BH}]$ a été interprété comme une suite de trois tons, /BHB/ avec un ton flottant bas final; [BB] comme une suite de deux tons; / $\mathrm{BH} /$ avec un ton haut flottant final; [HD] comme une suite de trois tons $/ \mathrm{HBH} /$, les deux derniers tons, bas et haut, étant flottants; et enfin $[\mathrm{HH}]$, comme un schème regroupant deux types de constituants: les constituants $[\mathrm{HH}]$-premier groupe pho- 
nologiquement structurés comme une suite de deux tons, $/ \mathrm{H} \underline{B} /$, dans lequel le dernier ton est flottant, et les constituants $[\mathrm{HH}]$-deuxième groupe phonologiquement analysés comme une suite de deux tons, /BH/, dans lequel il n'y a pas de ton flottant (se reporter à P.-A. Somé [1995] pour plus de discussion).

A la suite de ce rappel, le problème à traiter dans cet article, qui se concentre essentiellement sur le parler wule, concerne les tons flottants bas rencontrés dans les structures phonologiques $/ \mathrm{BH} \underline{B} /$ provenant de $[\mathrm{BH}], / \mathrm{H} \underline{B} /$ issu de $\mathrm{HH}$-premier groupe, et le ton flottant bas en position médiane dans $/ \mathrm{H} \underline{\mathrm{B}} \mathrm{H} /$ venant du schème phonétique [HD]. Autour de ces tons flottants bas, nous voulons défendre deux thèses: d'abord, montrer qu'en dagara les tons flottants bas, dans leur indépendance d'action [Odden 1995], sont capables de bloquer la propagation du ton haut précédent. Et pourtant l'expérience du wobe [Paradis 1984], par exemple, nous enseigne qu'un ton flottant ne peut pas bloquer le processus de propagation, car théoriquement un ton flottant doit s'associer ou disparaître. En wule, c'est l'inverse: un ton flottant bas peut non seulement bloquer le processus de propagation, mais aussi se maintenir dans le système. Or, on sait aussi que les consonnes opaques [cf. P. A. Somé 1998] bloquent également la propagation du ton haut précédent dans un contexte identique. Ces deux faits linguistiques nous conduisent à la deuxième thèse: au plan théorique, nous voulons montrer que, malgré la similarité des faits générés, à savoir le blocage du ton haut précédent par les tons flottants bas et par les consonnes opaques, il n'existe aucun lien de près ou de loin entre ces deux entités linguistiques.

Pour analyser ces faits, nous présentons, d'abord, sous la rubrique "remarques préalables" des données générales sur la langue. Ensuite nous allons montrer, dans une analyse nouvelle par rapport à l'état de connaissance de la langue, que les tons flottants bas existent de façon généralisée à l'instar du dschang-bamileke [Tadadjeu 1974], en nous servant de la combinatoire. Grâce à celle-ci, on sait que lorsqu'un ton haut est séparé d'un autre ton haut par un ton bas-flottant ou pas, le deuxième ton haut s'abaisse de niveau et génère un downstep.

A la suite de l'analyse sur les tons flottants, nous allons tenter de répondre à la question suivante: tout ton flottant bas autorise-t-il la propagation du ton haut précédent? Pour cela, nous allons encore utiliser la combinatoire pour montrer que, si tous les tons flottants bas sont des tons fixes, la différence entre eux réside dans le fait que certains d'entre eux en "position interne" (à définir plus loin) sont opposés à d'autres qui, eux, sont en "position externe" ou "périphérique" du fait qu'ils ont été re-assignés à un nouveau porteur. Nous allons, par la même occasion, montrer que le premier type de tons flottants peut, à l'instar des consonnes opaques [P.-A. Somé 1998], faire barrage à la propagation du ton haut de X1 sur l'unité suivante à ton bas, soit X2.

Pour terminer, nous allons répondre à une autre question: y a-t-il un lien entre ces tons flottants bas qui bloquent le ton haut précédent et les consonnes opaques qui, elles aussi, bloquent le ton haut précédent? Si oui, lequel?

Comme cadre théorique, nous allons utiliser la théorie autosegmentale [Goldsmith 1976; Williams 1976; Leben 1973] et confronter les données de la théorie aux faits du dagara. Nous allons en particulier confronter les faits du dagara aux 
exigences de "la règle de bonne formation" (the well-formedness condition) formulée comme suit:

(1) Règle de bonne formation:

a) Toutes les voyelles sont associées à au moins un ton.

b) Tous les tons sont associés à au moins une voyelle.

c) Les traits d'association ne doivent pas se croiser.

Plus loin, on montrera qu'il faudrait postuler d'abord, dans le cas du dagara, une règle, "a feeding rule" [O'Grady \& Dobrovosky 1992], qui pourra créer les condi-tions nécessaires à partir desquelles la règle de bonne formation peut s'appliquer.

\section{Remarques préalables}

Nous privilégions ici la discussion des tons au détriment de celle des voyelles et des consonnes du dagara. Pour plus d'information sur ces questions, voir P.-A. Somé [1982, 1989a, b, 1995, 1996, 1998].

2.1 Le système tonal. Le dagara a un système à deux tons: un ton haut $/$ ' $/$ et un ton bas $\Gamma /$, puis un downstep [P.-A. Somé 1982], dont la réalisation est provoquée par l'abaissement d'un ton haut par un ton bas réalisé ou flottant (voir sur ce point Stewart [1983]). Il peut être actualisé à l'intérieur ou entre les constituants. Il est représenté par le symbole l'/ indiquant le changement de niveau tonal. La distribution de ces deux tons est organisée par des règles de polarité tonale que l'on retrouve dans les langues voltaïques, notamment en lama, en moore [Kenstowicz 1988; Peterson 1971] ainsi qu'en lobr, l'une des variété dagara [Delplanque 1983, 1986], et dont l'application permet de dégager les principes ou axiomes suivants:

1) Tout segment dans le rôle du dérivatif, du suffixe de classe ou marque verbale, n'a pas de ton propre comme c'est le cas dans certaines langues africaines où ces morphèmes ont des tons fixes, toujours haut ou toujours bas. En dagara, ${ }^{1}$ c'est à partir du type de ton de la base ou du ton précédent que se décide, au niveau phonologique, le ton qui, dans ce cas de figure est toujours flottant. Ainsi, si la base porte un ton bas comme dans [zìlè] 'langues', le suffixe aura un ton haut phonologique flottant, soit /zìl-ź/; si par contre la base porte un ton haut comme dans [?yíilé] 'cornes', il aura un ton bas phono-

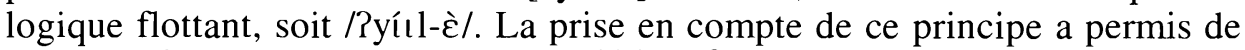
proposer l'illustration donnée sur les dérivatifs (5), les morphèmes verbaux (6), et les suffixes de classe (7).

2) Il arrive qu'une règle, celle d'assimilation tonale, voile la règle de polarité tonale. On rencontre cela lorsque le ton de la base, haut ou bas, s'impose au

\footnotetext{
'Nous voulons ici faire ressortir l'idée que le ton du suffixe ou du dérivatifs n'est pas connu d'avance. Il varie en fonction de la nature du ton précédent.
} 
domaine de réalisation du morphème suffixal: si la règle de polarisation fait que /zìlé/ porte un ton haut dans le suffixe, la règle d'assimilation tonale, elle, fait que le ton haut du suffixe cède sa place au ton bas de la base qui la repousse hors du cadre, d'où [zìlè]. C'est aussi le cas par exemple des constituants comme [níbé] qui vient de /níb-è/ 'personne', [búúlú] qui vient de /búul-ù/ 'bouillie aqueuse', [wààrà] qui vient de /wàa-dá/ 'sois en train de venir', [túúró] qui vient de /túu-dò/ 'sois en train de creuser' [P.-A. Somé 1982:221], dans lesquels tous les tons suffixaux sont flottants en dépit de la présence des suffixes vocaliques.

3) Pour les raisons qui précèdent, il faut bien souligner l'importance des tons flottants dans la langue, la définition "ton flottant" devant être comprise dans le sens de ton non réalisé explicitement mais provenant originellement des dérivatifs et/ou des suffixes qui se sont dissociés de leur ton parce qu'ils ont été reinterprétés comme segments faisant partie de la base. Les tons flottants ne sont donc pas des tons ajoutés pour les besoins de la description, mais des tons réels qui existent. Par ailleurs, les tons en dagara ne sont pas flottants parce qu'ils sont instables, mais plutôt parce qu'ils ont perdu leur support originel: en effet, tous les tons flottants sont très stables et sont rattachés à un support précis et fixe. Si nous avons choisi d'examiner de plus près, en wule, ces tons flottants bas c'est parce que, dans la combinatoire, ils déploient un dynamisme [Kinda 1983], et qu'à l'inverse, les tons flottants hauts qui se comportent comme des tons hauts réalisés n'offrent rien de particulier: en effet, les tons flottants hauts se propagent comme les tons hauts réalisés, et se trouvent, comme eux, bloqués dans le même contexte. ${ }^{2}$ On peut établir une comparaison entre les données de

2

a) le ton flottant haut dans les constituants se propage sur l'unité suivante comme le ton haut réalisé le fait, et n'est en rien différent de lui. En effet, si l'on considère les constituants [gìl] qui vient de /gìl-ǿ/ 'balafon' et [kùr] qui vient de /kùd-ǿ/ 'tortue', tous marqués par un ton flottant haut associé à une voyelle disparue symbolisée par $/ \phi /$, et qu' on leur associe respectivement des mots à ton bas dont les consonnes initiales sont transparentes, soient [tî́] $</$ t̀̀ın- $\hat{\emptyset} /$ 'médicament' et [mà] $</$ mà- $\phi \mid$ 'mère', eux aussi marqués par des tons flottants hauts, on obtient les suc-

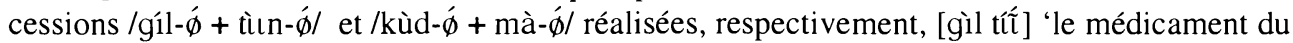
balafon' et [kìr má] 'la mère de la tortue'. Dans les deux cas, le ton haut flottant se propage sur le domaine du ton du deuxième constituant tout comme le fait le ton haut réalisé en (8a).

b) Le ton flottant haut peut être aussi bloqué par les consonnes opaques tout comme le ton haut réalisé (voir $8 \mathrm{~b}$ ). En effet, si l'on introduit les mêmes mots dans un contexte où le deuxième consti-tuant à ton bas est par ailleurs initié par une consonne opaque, respectivement [dì̀] 'case' et [bj̀w] 'trou', on voit que /gìl- $\hat{\phi}+$ diè/ est prononcé [gîl diè] 'la case du balafon', et /kùd- $\hat{\phi}$ + bj̀g/ est prononcé [kùr bj̀w] 'le trou de la tortue'. On obtient par ailleurs le même phénomène de blocage tonal si l'on remplace respectivement les constituants nominaux diè et bj̀w par une base verbale, la base dì qui signifie 'brûle'. Pour rendre la phrase acceptable, nous allons faire précéder les deux constituants /gìl- $\not /$ et /kùd- $\phi$ / par le mot [kà] qui signifie 'et'. Ainsi /kà + gìl$\grave{\phi}+$ dil$_{1}$ est prononcé [kà gìl di] 'et le balafon brûle', et /kà + kùd- $\phi+$ dì est prononcé [kà kùr dì] 'et la tortue brûle'. Ainsi, le point important à souligner ici est que, lorsque le ton haut flottant 
la note (1) et celles établies avec les tons hauts réalisés présentées plus loin (cf. $8 a)$.

Il importe de souligner, par ailleurs, que dans le processus de remorphologisation des langues voltaïques [Manessy 1969, 1975], parmi lesquelles le dagara, certains constituants syntaxiques ont produit de nouveaux suffixes pendant que d'autres ont perdu leurs suffixes de classe tout en conservant les tons. Cela a entraîné, en dagara, une absence de tons contraires sur -VV-, de sorte que *-V́V̀- et *-V̀V́-n'existent pas de façon explicite. La déduction importante est que l'unité porteuse de ton n'est pas la syllabe, mais plutôt le morphème, lequel peut avoir deux voyelles avec un seul ton, à l'exemple de [fáá] qui vient de /fáa-à/ 'retire un objet', [fá?] qui vient de /fáa/ 'retire plusieurs objets', [tú?] qui vient de /túu/ 'creuse', ou une seule voyelle avec un ton à l'exemple de [tá?] qui vient de /tád/ 'arrive'. Ainsi, si l'on dit que toutes les voyelles sont associées à au moins un ton (well-formedness condition), il faut spécifier que ce type d'association s'effectue au niveau phonétique en dagara. Ceci est d'autant plus vrai que, comme on l'a déjà affirmé, le système tonal dagara opère au niveau du morphème, et non au niveau de la syllabe.

2.2 Les notions de constituant syntaxique, de base, de dérivatif et de nominant/verbant en rapport au ton. Le constituant syntaxique défini comme l'unité minimale constitutive de l'énoncé [Houis 1980:10] est formé d'un lexème, de ndérivatifs et d'un nominant ou d'un verbant [Houis 1977, 1981, 1983], nominant et verbant ayant ici le sens de suffixe nominal ou verbal. Le schéma est celui dans (2).

(2) Constituant Syntaxique:

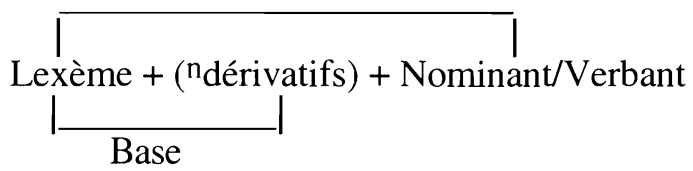

2.2.1 Le constituant syntaxique. Ainsi, les noms comme [kpó]/[kpór] trace(s)', [nír]/[níbé] 'personne(s), [wíć]/[wíír] 'sifflet(s)', [pàlá]/[pàlí], 'passage(s), [fíul'á]/ [fícl'í] 'petit(s) passages(s)', d'un côté, et les formes verbales comme [dirè] 'sois en train de manger', [(v) dirèná] '(il/elle) est en train de manger', [(v) diná] '(il/elle) a mangé', de l'autre, sont tous des constituants syntaxiques, respectivement nominaux et verbaux. Tout constituant syntaxique porte deux ou trois tons: deux tons répartis entre la base et le suffixe ([dìrè] pour /dìd-dé/); et trois tons lorsque la base a un deuxième ton, celui imparti au dérivatif ([fííl'á] pour /fítl-á/). De cette situation on peut déduire qu'en dagara à un morphème correspond toujours un ton.

2.2.2 La base. Il faut cependant souligner que si l'on peut appliquer ce schéma cidessus aux langues bantoues (swahili), ouest atlantiques (fulfulde), etc., où les

est bloqué par une consonne transparente, il se réalise sur n'importe quel support disponible de l'unité à laquelle il appartient. 
lexèmes sont séparables des dérivatifs, en dagara cela n'est pas possible: les structures les plus simples, même de type $\mathrm{CV}$, sont des unités complexes, comme en (3), dans lesquelles lexème et dérivatif sont imbriqués au point qu'il devient impossible de les séparer (voir Nikiéma [1989] sur la même question en moore, et Bonvini [1988] dans le cas du kasım). C'est pourquoi nous utilisons ici le concept de "base" au lieu de "lexème".

[dé] $<$ /déed/ 'prends'
[né] $<$ /néd/ 'vois habituellement'

En dagara on distingue deux types de base: les bases à un ton (4a) et celles à deux tons (4b). Pour plus de discussion voir P.-A. Somé [1996]. Il faut cependant souligner ici que toutes les bases à un ou à deux tons sont des bases dérivées, c'està-dire des bases complexes impliquant toutes des dérivatifs ayant un ton ou sans ton. Voici une illustration des deux types de base dans lesquels les consonnes (-d, - $-\mathbf{t},-\grave{n}$, $-l,-\grave{b},-\grave{g}$ ) et les voyelles (-à, -ì) sont des dérivatifs ayant des tons flottants bas, et dans lesquels on voit que le ton des dérivatifs est l'inverse de celui des bases.

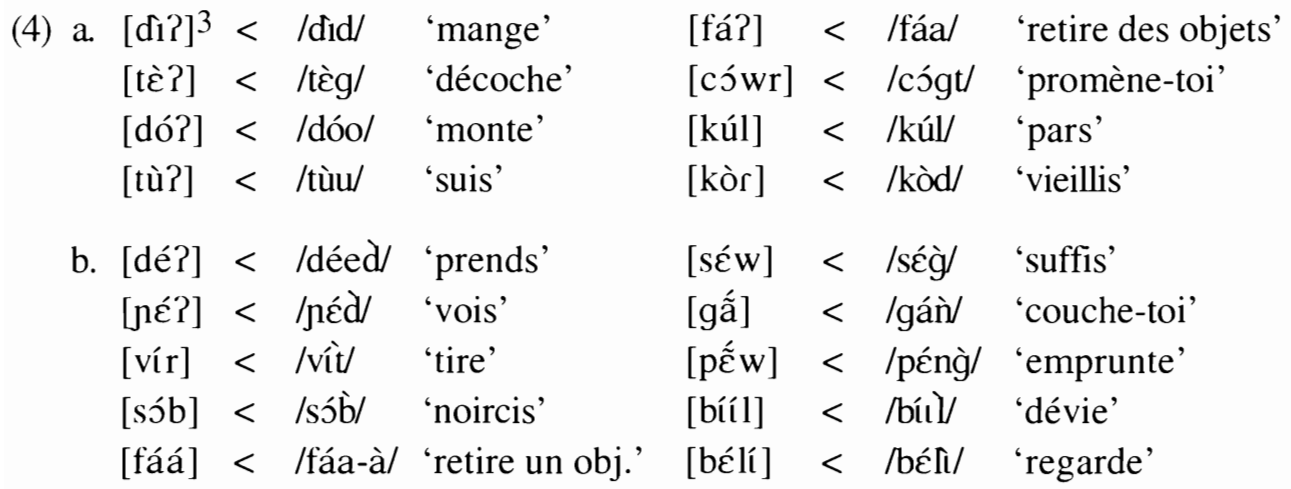

2.2.3 Les dérivatifs. Le dagara n'a pas de morphèmes dérivatifs pré-établis. Comme on l'a vu plus haut, une consonne ou une voyelle peut être re-interprétée

\footnotetext{
3 Les bases de type CV sont prononcées avec un coup de glotte, soit [CV?], qui renvoie toujours du point de vue morphologique à un segment / $-\mathrm{b},-\mathrm{d},-\mathrm{g} /$ ou à un segment vocalique de même timbre que la première voyelle de la base tel que dans [tú?] </túu/ 'creuse', et quelquefois à tout un ensemble de segments, /-ed/ dans [dé?] </déed/ 'prends', par exemple. Pour respecter la morphologie de la langue et l'intégrer dans l'analyse, nous avons décidé de reproduire ces données sous-jacentes; les barres obliques que nous utilisons ne détruisent en rien l'idée que ces éléments sous-jacents sont d'ordre morphologique. Plus loin, nous verrons que les barres obliques sont utilisées pour les tons phonologiques, les barres parallèles pour les tons morphologiques et les crochets pour les tons phonétiques: nous n'avons pas jugé utile d'utiliser les barres parallèles pour indiquer à la fois la structure morphologique des segments et le niveau morphologique des tons. L'analyse étant d'ordre tonal, nous avons exclusivement utilisé les barres parallèles du niveau morphologique pour faire ressortir la différence entre les trois niveaux d'analyse tonale.
} 
comme dérivatif aujourd'hui insécable de la base, comme en Kasım [Bonvini 1988]. Il découle par ailleurs du principe de polarité tonale que le ton du dérivatif est l'inverse de celui de la base:

i) Dans les exemples de (5a), les bases verbales de type [CV] et [CVV], font apparaitre respectivement une voyelle $-\mathrm{V}$ (en position finale) et - $\mathrm{V}$ - (en position médiane) qui sont des voyelles géminées re-interprétées comme dérivatifs. Par ailleurs, dans les bases où il y a plusieurs dérivatifs, seul le dernier dérivatif génère un ton. On remarquera enfin que dans les bases à ton bas il n'y a pas de ton flottant haut (pour plus de discussion voir P.-A. Somé [1996]).

(5) a. Morphèmes dérivatifs: $-\mathrm{a},-\mathrm{o},-\varepsilon,-\mathrm{u},-\mathrm{-}$.

Base de type [CV]

[dá?] < /dáa/ 'pousse des choses'

[fó?] < /fóo/ 'extrais des choses'

[zغ̇̀] < /zع́ع/ 'pillez des choses'

[gò?] < /gòs/ 'tiens droit les

parties du corps'
Base de type $[\mathrm{CVV}]$

[dáá] < /dáa-à/ 'pousse une chose'

[fúó] < /fóo-ò/ 'extrais une chose'

[ż̀̀] $</ z \varepsilon ́ \varepsilon-\varepsilon / 4$ 'retire par force une chose'

[gùò] < /gòo-o/ 'courbe une partie du corps'

ii) Dans la nominalisation des bases verbales de type CV (5b), -V- est une voyelle géminée re-interprétée comme dérivatif.

(5) b. Au niveau du mot

Bases verbales

[dé?] 'prends'

[di?] 'mange'

[né?] 'vois'

[lò?] 'tombe'
Morphèmes dérivatifs: -e-, -i-, - $\varepsilon-,-$ a-

Verbes transformés en noms

[dé'éb] < /dé-è-b/ 'fait de prendre'

[dííb] </di-i-b/ 'fait de manger'

[ní'ćb] < /nć-è-b/ 'fait de voir habituellement'

[ló-a-b] < /lò-a-b/ 'fait de tomber'

iii) A partir des bases de type $\mathrm{CV}$, on peut encore dériver d'autres bases ayant soit une structure de type CVC ou CVCC avec -b, -d, -n, -t, -l comme dérivatifs (5c), soit une structure de type CVVC avec $-\mathrm{t},-1,-\mathrm{n}$ comme dérivatifs (5d).

4 En wule, toutes les bases à ton bas de type CV comme [zغ̀?] $</$ zèc/ 'pillez plusieurs choses',

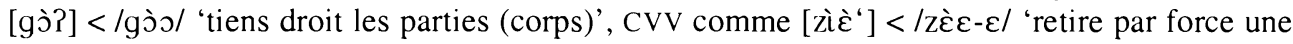
chose', [gù̀̀]: /gòo-J/ 'courbe une partie (corps)', ou même CVC comme [?ir] </Rìt/ 'lève-toi', n'ont pas de ton sur le dérivatif. En birfuor, par contre, on distingue deux groupes de verbes dans ces types de structure: les verbes à ton bas qui n'ont pas de ton de dérivatif commme [gbà?]< /gbàt/, ì gbàràná 'je joue aux quatre cauris', [gbòw]</gbòg/, ĩ gbòwròná 'j'allume', et ceux à ton bas dont le dérivatif est marqué par un ton flottant: c'est le cas de [nmè?] </nmè-é/, ì nmèèréná 'je suis en train de frapper', [nmàà] </ymà-á/, ì nmààráná 'je suis en train de couper une fois', [dà?] </dà-á/, î dààráná 'je suis en train d'acheter'. 
(5)

c. [fó?] 'extrais des saletés' .[fób] /fób/ 'extrais en perforant avec le bec'

.[fór] /fód/ 'détache une goutte avec les lèvres à partir d'une calebasse'

[fá?] 'retire des objets' .[fár] /fád/ 'rase tous les cheveux'

.[fán] /fánǹ/ 'extrais des idées d'une réflexion (pense)'

[dá?] 'pousse des objets' .[dár] /dát/ 'va ici et là (de façon désordonnée)'

[wé?] 'pourris' .[wél] /wél/ 'désintègre-toi (à la suite d'une pourriture)'

d. [fá?] 'retire des objets' .[fáár] /fáat/ 'mets-toi entre deux personnes pour les empêcher de se battre'

[dá?] 'pousse des objets' .[dáal] /dáal/ 'pousse un objet avec de l'eau (mets beaucoup d'eau dans la sauce)'

[wé?] 'pourris'

.[wícl] /wíll/ 'sois sans utilité '

.[wíć] /wícǹ/ 'viens au secours de'

2.2.4 Le nominant/verbant ou suffixe nominal/suffixe verbal. Les suffixes de classe (nominants) et les suffixes verbaux (verbants) dont les tons sont déterminés par les tons des bases s'organisent comme suit:

2.2.5 Les suffixes verbaux. Deux morphèmes méritent d'être signalés, $|\mathrm{dV}|$ qui marque la progression de l'action et dont le ton est déterminé par le dernier ton de la base, et |na| toujours associé aux verbes conjugués et dont le ton est déterminé soit par celui de $|\mathrm{dV}|$, soit par celui du morphème tonal (cf. P.-A. Somé [1982 :223]) qui marque l'aspect accompli. En voici, dans (6), une illustration.

(6) Forme inaccomplie

\begin{tabular}{|c|c|c|}
\hline [?] & /tád-dV̀-ná/5 > [(ò) táráná] & \\
\hline ?] 'vien & /wàa-dV́-nà/ > [(ì) wààràná] & t en train de venir' \\
\hline & $>$ [(è) túúróná $]$ & est en train \\
\hline 'mange/brûle' & $>$ [(ì) dirènà $]$ & est en train de manger' \\
\hline
\end{tabular}

5 Je voudrais encore souligner que dans les langues voltaïques, il n'y a pas toujours de correspondence entre les formes phonétiques et les formes phonologiques. C'est ainsi qu'en dagara les formes phonétiques des verbes de type CV à l'exemple de [tá], [wà] ou [tá?], [wà?], si l'on tient comme du coup de glotte démarquatif, ont comme formes phonologiques /tád/, /wáa/, etc., et non */tá/, */wà/. Les formes notées /tád/, /wáa/, etc. peuvent être en réalité considérées comme morphologiques. Cependant, pour mettre l'accent sur les tons et non sur les segments, nous avons préféré poser ces formes comme phonologiques et non morphologiques. 
Forme accomplie

\begin{tabular}{|c|c|c|c|}
\hline 'arr & /tád- & $>$ [(ì) tán'á] & ‘il/ell \\
\hline ] 'vie & /wàa-'-ná/ & > [(î̀) wàná] & 'il/elle est venu(e)' \\
\hline 'cre & /túu-’-ná/ & > [(è) tún'á] & 'il/elle a creusé' \\
\hline e/brûle' & /dıd-'-ná/ & $>$ [(ì) dìná $]$ & 'il/elle a mangé' \\
\hline
\end{tabular}

Forme injonctive non progressive

Prononcé:

Forme sous-jacente:

$\begin{array}{lll}\text { [tá?] 'arrive' } & \text { /tád/ } \\ \text { [wá?] 'viens' } & \text { /wàa/ } \\ \text { [tú?] 'creuse' } & \text { /túu/ } \\ \text { [dí?] 'mange' } & \text { /did/ }\end{array}$

Forme injonctive progressive

\begin{tabular}{|c|c|c|c|c|}
\hline tá? & 'arrive' & /tád-dỳ/ & $>$ [tárá] & 'sois en train d'arriver' \\
\hline & 'viens' & /wàa-dv́/ & $>$ [wààrà] & ain de venir' \\
\hline [tú? & 'creuse' & /túu-dv̀/ & $>$ [túúró] & ain de creuser' \\
\hline fi?] & 'mange/brûle' & /did-dV́/ & $>$ [dirè $]$ & de manger' \\
\hline
\end{tabular}

2.2.6 Les suffixes nominaux. En (7) se trouve la liste complète des suffixes de classe en wule répartie en singulier et pluriel et dans laquelle, rappelons-le, le signe $/ \phi /$ représente une voyelle ou un suffixe de type $\mathrm{CV}$ disparu(e). Les barres parallèles indiquent qu'il s'agit du niveau morphologique où les morphèmes ne portent pas de ton, tandis que les barres obliques indiquent le niveau phonologique où l'association des tons aux morphèmes a lieu. Ces classes nominales s'illustrent dans les constituants comme suit (voir aussi Delplanque [1979, 1983, 1986]).

2.3 Consonnes opaques versus consonnes transparentes. L'idée centrale autour de ces consonnes est d'affirmer que les consonnes transparentes favorisent la propagation du ton haut précédent alors que les consonnes opaques la bloquent (voir Hyman [1973, 1974] et Tchagbale [1984, 1989] sur la même question). En effet, dans P.-A. Somé [1998], nous avons montré que les consonnes transparentes-p, $\mathrm{f}, \mathrm{t}, \mathrm{s}, \mathrm{c}, \mathrm{k}, \mathrm{kp}, \mathrm{h}, \mathrm{?}, \mathrm{m}, \int, \mathrm{n}, \mathrm{l}, \mathrm{?}, \mathrm{l}, \mathrm{n}, \mathrm{y}, \mathrm{y}, \mathrm{N}, \mathrm{w}, \mathrm{w}, \mathrm{nm}$-favorisent la propagation du ton haut précédent, celui de X1 sur la première syllabe à ton bas de $\mathrm{X} 2$, X1 et $\mathrm{X} 2$ représentant chacun les mots dans l'ordre qu'ils décrivent. On peut le voir ici dans les exemples dans (8): les bases verbales en X2 que sont, par exemple, [pù], ${ }^{6}$ [fà], [tì], [sò], [cì], [kà], [kpà], [hèli],ont leur ton bas délogé et maintenu flottant par le ton haut propagé de Xl représenté ici par la base verbale [tá] 'arrive' citée en (6). On peut aussi voir dans (8b) que les consonnes opaques-b, v, d, z, j, g, gb,

${ }^{6}$ C'est volontairement que nous ne tiendrons plus compte de la consonne glottale démarcative en fin des formes verbales de type CV. Elle n'intervient en rien dans la démonstration qui nous préoccupe. 
(7) Singulier

$$
\begin{aligned}
& \text {-d: [nír] < /ní-dǿ/ } \\
& \text { [kpéw'í] < /kpég̀-dø/ / }
\end{aligned}
$$

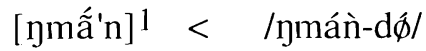

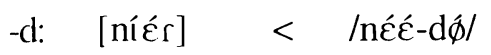

$$
\begin{aligned}
& \text { [yúór] < /yòo-dø̄/ } \\
& \text {-A: [fáá] < /fáa-à/ } \\
& \text { [láá] < /làa-á/ } \\
& \text { [gbìè] < /gbè-á/ }
\end{aligned}
$$

-A: [górá] < /gòd-ál

[sìlà] < /s̀̀l-ál

[fíil'á] < /fúulá/

-g/ф: [zàg] < /zà-gø/

[wáb] < /wáb-ф/

-E: [pùrè] < /pùd-é/

[búlé] < /bùl-é/

[gbélé] < /gbél-غ̀l

\begin{tabular}{|c|c|c|c|c|}
\hline -bV: & $\begin{array}{l}\text { [níbé] } \\
\text { [kpébé] }\end{array}$ & $\begin{array}{l}< \\
<\end{array}$ & $\begin{array}{l}\text { /ní-bè/ } \\
\text { /kpég-bè/ }\end{array}$ & $\begin{array}{l}\text { 'personne(s)' } \\
\text { 'gésier(s)' }\end{array}$ \\
\hline & [Đmámá] & $<$ & /ṇmán-bà/ & 'tourterelle(s)' \\
\hline$-E:$ & [néć] & $<$ & /nع́ع-غ̀/ & 'meule(s) traditionnelle(s)' \\
\hline & [ywéé] & $<$ & /yòo-é/ & 'gros canari(s)' \\
\hline$-\mathrm{E}:$ & [féć] & $<$ & /fáa-è/ & 'pigeon(s) vert(s)' \\
\hline & [léć] & $<$ & /làa-ć/ & 'jarre(s)' \\
\hline & [gbèè] & $<$ & /gbè-é/ & 'front(s)' \\
\hline$-d v:$ & [górí] & $<$ & /gòd-dí/ & 'chambrette(s)' \\
\hline & [sìlì ] & $<$ & /sìl-dí/ & ‘épervier(s)’ \\
\hline & [fúıl’í] & $<$ & /f̌ùldí/ & 'petit(s) passage(s)' \\
\hline$-\mathrm{t}:$ & [zàr] & $<$ & /zâ-tǿ / & 'entrée(s) de maison(s)' \\
\hline & [wáwr] & $<$ & /wáb-tф/ & 'éléphant(s)’ \\
\hline$-\mathrm{dV}:$ & [pùrì] & $<$ & /pùd-di/ & 'tante(s)' \\
\hline & [búlí] & $<$ & /bùl-di/ & ‘puits’ \\
\hline & [gbélí] & $<$ & /gbél-di/ & ‘épis de mil’ \\
\hline & [kpàrí] & $<$ & /kpàd-dî/ & 'habit(s)' \\
\hline & [tùlí] & $<$ & /tùl-di// & 'pilon(s)' \\
\hline
\end{tabular}

-U: [kpàrú] < /kpàd-ò/

[tùlú] < /tùl-ù/

-b: [dííb] $<$ /dúi-b/ 'fait de manger'

\section{Pluriel}

'fait de creuser'

${ }^{1}$ Il faut remarquer dès à présent que le downstep qui apparaît dans [nmấ' ń] au singulier disparaît dans [ĐĐmámá] au pluriel, et qu’il est maintenu aussi bien au singulier qu'au pluriel des mots comme [fí́lá] / [fí́lí] dans la liste ci-dessus. Cette différence servira à expliquer plus loin que dans le premier cas, le downstep est lexicalisé alors qu'il ne l'est pas dans le second cas. 
(8) a. tá + pù tá + fà

tá + tì

tá + sò

tá + cì

tá + kà

tá + kpà

tá + hèlı

b. tá + bù

tá + và

tá + tì

tá + zò

tá + jì

tá + gà

tá + gbà

tá + hèlì devient

/tá pû/ puis
/tá fá/ "
/tá tî/ "
/tá sô/ "
/tá cî/ "
/tá kâ/ "
/tá kpâ/ "
/tá héீ1/ "

devient

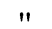

/bá bù/ puis
/bá và/ "
/bá tı/ "
/bá zò/ "
/bá ji// "
/bá gà/ "
/bá gbà/ "
/bá hèlı/ "

[tá pú]

[tá fá]

[tá tí]

[tá só]

[tá cí]

[tá ká]

[tá kpá]

[tá héli] 'arrive et fleuris!'

'arrive et sois foutu!'

'arrive et vomis!'

'arrive et épice!'

'arrive et parie!'

'arrive et casse!'

'arrive et cloue!'

'arrive et éclate de rire!'

[bá bù] 'arrive et mouille!'

[bá và] 'arrive et bats (mil)!'

[bá tì] 'arrive et mange!'

[bá zò] 'arrive et cours!'

[bá jı] 'arrive et taille!'

[bá gà] 'arrive et grignote!'

[bá gbà] 'arrive et joue!'

[bá hèI1] 'arrive et élargis!'

h-en position initiale des bases à ton bas comme [bà], [và],[dî], [zò], [j̄i ], [gà], [gbà], [hè 1 ], bloquent au contraire la propagation du ton haut de X1 toujours porté par la base verbale [tá] 'arrive'. De cette situation, nous dégageons la règle suivante:

RÈGLE: Tout ton $\mathrm{H}$ précédent se propage sur le domaine de la syllabe à ton bas suivante si cette syllabe est initiée par une consonne transparente, il est par contre bloqué si la syllabe suivante est initiée par une consonne opaque.

Il y a deux exceptions:

1) Le ton $\mathrm{H}$ se propage sur tout pronom personnel complément, que celui-ci soit constitué de voyelles simples ([à] 'pronom personnel, troisième personne du pluriel des choses', [ì] 'pronom personnel troisième personne, singulier)' ou de structure CV dans laquelle [c-] est, soit une consonne opaque (p. e. [bà] 'pronom personnel troisième personne du pluriel'), soit une consonne transparente (p. e. [tì] 'pronom personnel première personne du pluriel', [yغ̀]: 'pronom personnel deuxième personne du pluriel'). Ainsi, si l'on fait suivre par exemple le verbe [tú] 'creuse' donné en (6) par les pronoms personnels, on obient les formes dans (8c).
(8) c. tú + ̀̀
est prononcé
[tú ú]
'creuse-le'
tú + à
[tú á]
'creuse-les'
tú + tì
[tú tí]
'creuse-nous'
tú + yè
[tú yé]
'(qu'il / elle) vous creuse'
tú + bà
[tú bá]
'creuse-les' 
2) Il n'y a que le ton $\mathrm{H}$ qui se propage dans les conditions ci-dessus décrites, mais pas le ton bas. En effet, le ton bas en dagara ne se propage normalement que dans le cadre de mots composés figés. En voici une illustration (8d), dans laquelle les bases *[sĩ] et *[tà] qui ne s'emploient plus seules comme mots isolés, et [gbúló] 'gros et ronds', et [kórí] 'ancien', des adjectifs ayant des tons hauts en mots isolés, sont mis en rapport d'association. On constate que le ton bas des bases se propage et s'impose non pas seulement sur la première syllabe du mot suivant comme dans le cas du ton haut, mais sur l'ensemble du mot suivant.

(8) d. sĩ̀ + gbúló est prononcé tà + kórí [sĩ gbùlò] 'petits poids (de terre)'

[tà kòrì] 'le temps des ancêtres'

Notre analyse ici ne s'occupera que de la propagation du ton haut précédent. Pour cela, nous allons, d'abord, identifier dans la position de X1 les schèmes ayant un ton flottant bas final comme dans $/ \mathrm{H} \underline{\mathrm{B}} /, \mathrm{BH} \underline{\mathrm{B}} /$ ainsi que dans $/ \mathrm{H} \underline{\mathrm{B}} \mathrm{H} / \mathrm{dont}$ le deuxième ton est flottant tout comme le ton final; ensuite, nous allons les mettre en rapport avec X2, c'est-à-dire, des bases ou des constituants dont la première syllabe est à ton bas et les consonnes initiales transparentes. La prise en compte des consonnes transparentes en position initiale de X2, à l'exclusion des consonnes opaques, permettra de clarifier ceci : si vraiment il y a une action de blocage, le ton haut précédent ne pourra effectivement pas se propager sur X2 en dépit de la présence des consonnes transparentes en X2. Par contre, si le ton bas en question n'a pas la capacité de bloquer le ton haut précédent, celui-ci va se propager sur X2.

Nous allons à présent examiner les points respectifs de notre plan en gardant à l'esprit les faits suivants:

i) Il sera chaque fois nécessaire de prouver la présence du ton flottant bas de X1 par l'usage de la combinatoire et de motiver le X2 en prenant comme exemples leurs formes en mots isolés.

ii) Pour l'association de $\mathrm{X} 1$ à deux tons avec $\mathrm{X} 2$, il faut toujours se rappeler que la consonne initiale de X2 est transparente. Ainsi, dans /ní-d $\varnothing /+/$ pùod/ 'le dos/ derrière de quelqu'un', la consonne initiale sur laquelle s'exercera l'influence du ton haut n'est pas le $n$ - de /ní-d $\phi /$, mais plutôt le $p$ - de /pùod- $\phi /$.

iii) Trois niveaux d'analyse, à savoir les niveaux morphologique ||, phonologique //, et phonétique [ ] sont généralement maintenus. Cependant, dans le présent article, nous utiliserons plus fréquemment les niveaux phonétique [ ] et phonologique / /.Les chiffres 1, 2, 3, etc., indiquent la dégradation des niveaux tonals, le chiffre 1 est le niveau le plus élevé, le chiffre 2, le niveau suivant, etc., tandis que le symbole [- ${ }^{-}$] est celui d'un ton haut abaissé, le symbole [- ${ }^{-}$, celui des tons hauts du même niveau; le signe < signifie 'se réécrit (forme phonologique)'; et le signe > signifie 'donne (forme phonétique)'. L'un et l'autre indiquent le passage d'un niveau à un autre. 


\section{Les tons flottants bas en dagara: dans quels types d'unités ?}

Les tons flottants bas existent dans toutes les unités lexicales de la langue. On les trouve aussi bien dans les constituants que dans les bases verbales et nominales. Nous allons tour à tour examiner chacune de ces unités avec comme objectif celui de faire ressortir la présence du ton flottant bas dans chaque cas.

\subsection{Les tons flottants bas dans les constituants.}

3.1.1 Les constituant nominaux. Ainsi que nous l'avons initialement indiqué, pour prouver l'existence des tons flottants bas il faut placer après une première unité à ton haut une deuxième à ton haut. Si le ton de cette deuxième unité n'est pas abaissé, c'est qu'il n'y a pas un ton flottant bas dans la première unité; et si le ton haut est abaissé c'est qu'il y a un ton flottant bas dans la première. Dans la comparaison suivante, on a d'un côté [bíé] 'enfant' et [pów] 'femme' qui constituent les premières unités en (9). On a de l'autre côté [bíé] 'jeux' et [nír] 'personne', les premières unités en (10). Toutes ces unités ont dans leur forme isolée des tons hauts et sont respectivement de type CVV et CVC. Plus loin on verra que [bíé] 'enfant' et [bíé] 'jeux' constituent des paires minimales au niveau tonal. Par ailleurs, les constituants [zú], [pié], [yír], [kúór], [kpấw], [níbé], [yébé], respectivement, sont de type $\mathrm{CV}, \mathrm{CVV}, \mathrm{CVC}, \mathrm{CVVC}, \mathrm{CVCC}, \mathrm{CVCV}$, utilisés comme deuxièmes unités, aussi bien en (9) qu'en (10), et sont également à ton haut en mot isolé. (On y remarquera que [bíé] 'enfant' est remplacé par [pów] 'femme' dans (9b).

(9)

\begin{tabular}{|c|c|c|c|c|}
\hline bíé + zú & prononcé & {$\left[\begin{array}{lll}b^{\prime} e_{1} & z u ́ \\
1\end{array}\right]$} & $\mathrm{CV}$ & 'la tête de l'enfant' \\
\hline bíé + píé & . & [bié $e_{1}$ piéé $\left._{1}\right]$ & $\mathrm{CVV}$ & 'les dix de l'enfant' \\
\hline bíé + yír & " & [bíé yír $\left._{1}\right]$ & $\mathrm{CVC}$ & 'la maison de l'enfant' \\
\hline bíé + kúór & " & [bíé kvúór $_{1}$ ] & CVVC & 'la gourde de l'enfant' \\
\hline bíé + kpấw7 & " & {$\left[\right.$ bié $_{1}$ kpấw $\left._{1}\right]$} & $\mathrm{CVCC}$ & 'la force de l'enfant' \\
\hline bié + níbé & " & [bíé ${ }_{1}$ níbé 1$]$ & $\mathrm{CVCV}$ & 'les parents de l'enfant' \\
\hline pów + píé & $\begin{array}{l}\text { onc } \\
\text { " }\end{array}$ & [pów píé $\left._{1}\right]$ & & a femme' \\
\hline
\end{tabular}

Le ton haut de la deuxième unité en (9) n'est pas abaissé; par contre, il est abaissé en (10). Si, dans le même contexte, l'on remplace [bíé] 'jeux' par [nír] 'personne' en (10b), le résultat reste le même. Remplaçons à présent le constituant [nír] de type CVC par [yébé] qui, lui, est de type CVCV; le résultat est le même en dépit de la présence du suffixe vocalique [-É] dans [-bé].

7 Plus loin, on verra que la forme kpấw vient en fait phonologiquement de /kpáng/, le tilde étant une réalisation de la nasale $/ \mathrm{n} /$. 


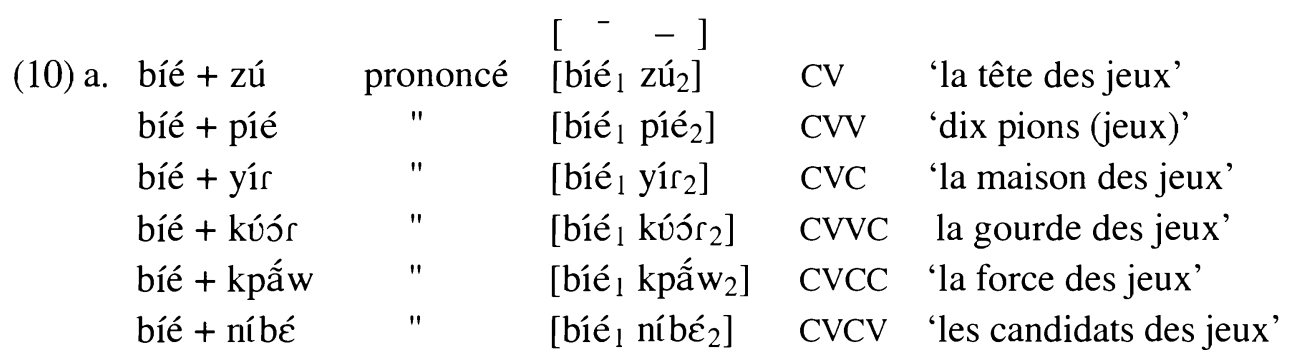

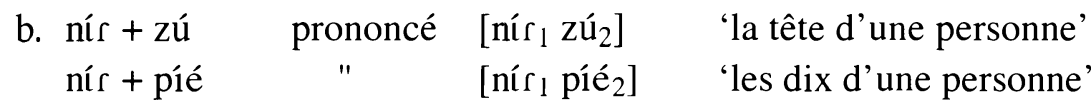

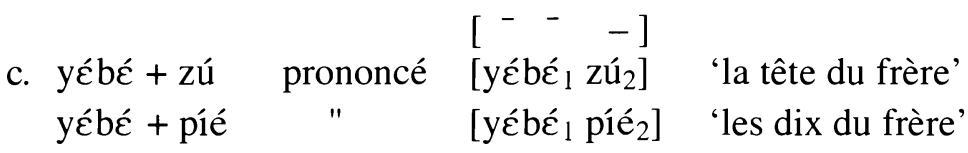

Ainsi, l'absence d'abaissement tonal en (9) fournit la preuve qu'il n'y a aucun ton flottant bas dans les premières unités: [bíé] et [pów]. En effet, ces deux constituants sont du schème tonal $[\mathrm{HH}]$-deuxième groupe dans lequel il n'y a pas de ton flottant bas. Le schème tonal est $/ \mathrm{BH} /$. Cela implique que les structures tonales phonologiques de ces constituants sont respectivement /bì-é/ et /pòg-ǿ/. Par contre, l'abaissement en (10) indique que [biéé] 'jeux', [nír] 'personne', [yébé] 'frère' et, avec eux, tous les constituants du même type, soient par exemples [léć], [nìd], [kál], [gbéd], [píír], [váár], [wáwr], ont tous deux tons, un ton haut et un ton flottant bas qui reste toujours flottant même en présence des suffixes vocaliques. Cela nous autorise à déduire les formes phonologiques citée dans (11).

\begin{tabular}{|c|c|c|c|c|c|c|}
\hline (11) [bíé] & $<$ & /bí-è/ & ou & /bíè/ & $\mathrm{CVV}$ & 'jeux' \\
\hline [nìd] & $<$ & /níd-фे/ & " & /níd/ & $\mathrm{CVC}$ & 'personne' \\
\hline [léć] & $<$ & /láa-غ̀/ & " & /láaغ̀/ & CVVV & 'haches' \\
\hline [kál] & $<$ & /kál- $\phi /$ & $"$ & /kál/ & & ‘épices’ \\
\hline [púír] & $<$ & /píi-tфф/ & $"$ & /píit/ & CVVC & 'moutons' \\
\hline [váár] & $<$ & /váa-dø/ & $"$ & /váad/ & & 'feuilles' \\
\hline [wáwr] & $<$ & /wáb-tø/ & $"$ & /wábì/ & CVCC & 'éléphants' \\
\hline [yébé] 8 & $<$ & /yع́b-غ̀/ & $"$ & /yćbè/ & $\mathrm{CVCV}$ & 'frère' \\
\hline
\end{tabular}

${ }^{8}$ Nous insiston ici sur le fait que le ton bas phonologique du constituant /yébè/ est flottant tout comme dans le cas de /bièlou de /níd/. La présence du ton flottant est une question de morphème et non de structure du mot. 
3.1.2 Les constituants verbaux. La situation est la même pour les constituants verbaux. Dans les exemples suivants, remplaçons [bíé] 'enfant' de l'exemple (9a) par [tá] ${ }^{9}$ 'arrive/approche', la forme isolée de l'injonctif non progressive dans l'exemple (12), et [bié] 'jeux' de l'exemple (10a) par [tárá], la forme isolée de l'injonctif progressif dans l'exemple (13a). Et puis, maintenons comme deuxièmes unités les mêmes constituants utilisés aussi bien en (9) qu'en (10). On obtient, ensuite, le résutat en (13a).

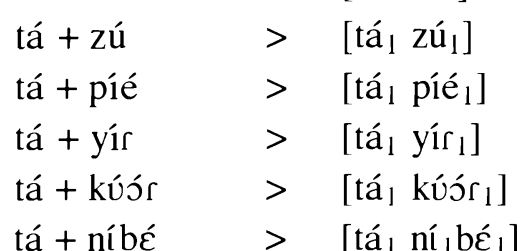

'approche-toi d'une tête'
'sois proche de dix'
'approche-toi d'une maison'
'approche-toi de la gourde'
'approche-toi des personnes' $\left[{ }^{--}-\right]$

(13) a.

\begin{tabular}{|c|c|c|}
\hline rá + zú & $>$ & [tárá ${ }_{1}$ zú 2 ] \\
\hline - píé & & [tárá píé$\left._{2}\right]$ \\
\hline $18-1$ & & [tárá 1 yír ${ }_{2}$ ] \\
\hline & & [tárá ${ }_{1}$ kúór 2 \\
\hline$+n$ & & [tárá ${ }_{1}$ níbé \\
\hline
\end{tabular}

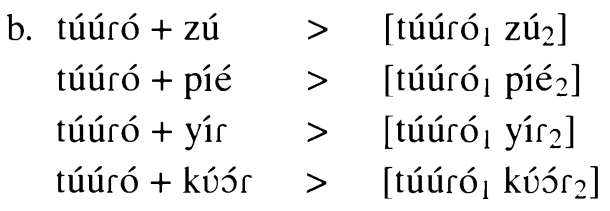

En comparant les deux résultats, soit (12) et (13) on s'aperçoit qu'il n'y a pas d'abaissement tonal en (12), ce qui est l'inverse en (13). En effet, le ton haut du constituant après [tárá] et [túúró] est abaissé, signe que ces deux constituants verbaux ont chacun un ton flottant bas final. Cela nous autorise à interpréter phonologiquement, d'une part, le mot [tá] et avec lui tous les autres mots du même type tels que, [tú], [kó], [kúl], [lób], [báw], [fówr], comme étant des mots à un ton haut simple (14a); et d'autre part, les constituants verbaux [tárá], [túúró] et, avec eux, tous les autres constituants verbaux du même type tels que [kúóró],

\footnotetext{
9 Il faut remarquer que la forme [tá] peut être considérée à la fois comme un constituant et une base verbale. Elle est considérée comme constituant si on la prend comme forme conjuguée de l'injonctif non progressif opposée comme telle à la forme [tárá] de l'injonctif progressif. Elle est considérée par ailleurs comme une base verbale si l'on considère que c'est cette forme qui doit être entrée dans le lexique pour traduire ce qui correspondrait à la forme infinitive du français 'arriver'.
} 
[kúló], [lówró], [báwró], [fówró], comme ayant deux tons, le deuxième ton, celui de /dV/, étant flottant bas (14b).

\begin{tabular}{|c|c|c|c|c|c|}
\hline \multirow[t]{7}{*}{ (14) a. } & [tá] & $<$ & /tád/ & $\mathrm{CVC}$ & 'arrive' \\
\hline & [tú] & $<$ & /túu/ & $\mathrm{CVV}$ & 'creuse' \\
\hline & [kó] & $<$ & /kóo/ & & 'cultive' \\
\hline & [kúl] & $<$ & /kúl/ & $\mathrm{CVC}$ & 'pars' \\
\hline & [lób] & $<$ & /lób/ & & 'lance' \\
\hline & [báw] & $<$ & /bág/ & & 'écrase' \\
\hline & [fówr] & $<$ & /fógt/ & CVCC & 'enlève' \\
\hline \multirow[t]{7}{*}{ b. } & [tárá] & $<$ & /tád-dà/ & & 'sois en train d'arriver' \\
\hline & [túúró] & $<$ & /túu-dò/ & & 'sois en train de creuser' \\
\hline & [kúór] & $<$ & /kóo- $\underline{\mathrm{dj}} /$ & & 'sois en train de cultiver' \\
\hline & [kúló] & $<$ & /kúl-dò/ & & 'sois en train de partir' \\
\hline & [lówró] & $<$ & /lób- $\underline{\mathrm{d} \partial} /$ & & 'sois en train de lancer' \\
\hline & [báwró] & $<$ & /bág-dذ̀/ & & 'sois en train d'écraser' \\
\hline & [fówró] & $<$ & /fóg-dò/ & & 'sois en train d'enlever' \\
\hline
\end{tabular}

En résumé, les exemples que nous venons d'examiner confirment qu'un certain type de constituants nominaux et verbaux génère automatiquement des tons flottants, lesquels manifestent leur présence en rabaissant le ton haut qui suit. Il nous faut à présent examiner le cas des bases verbales et des bases nominales.

3.2 Les tons flottants bas dans les bases verbales et nominales. Certaines bases verbales comme nominales sont marquées par un ton flottant bas. Nous allons essayer de les identifier pour pouvoir les distinguer plus loin; car, rappelons-le, tous les tons flottants bas ne déploient pas le même dynamisme dans la combinatoire.

3.2.1 Les tons flottants bas dans les bases verbales. On retrouve des tons flottants bas générés dans certaines bases verbales à ton haut. En effet, si on associe à ces bases, comme par exemple [dé] 'prends' dans sa forme isolée d'autres consti-tuants-tels que [zú], [píé], [yír], [kúór], [níbé], qui, comme on l'a vu en (9) et (10), sont à ton haut dans leurs formes isolées-le ton haut est abaissé. Comparons, par exemple, le résultat en (15a) avec celui obtenu avec [tá] 'arrive' en (12).

Comme on l'a initialement vu dans les exemples en (12), il n'y a pas d'abaissement tonal contrairement à ce qui est observé en (15a). Cela signifie que la base [dé] a un ton flottant bas. Toutefois, pour prouver que cette base n'est effectivement pas la seule à avoir un ton flottant bas, remplaçons-la dans le même contexte par une autre base verbale, la base [nów] 'attrape', une base à ton haut citée dans la liste ci-dessous (cf. 16). On obtient le même résultat qu'en (15a). 


\begin{tabular}{|c|c|c|c|}
\hline \multirow{7}{*}{$\begin{array}{l}\text { (15) a. dé + zú } \\
\text { dé + píé } \\
\text { dé + yír } \\
\text { dé + kúór } \\
\text { dé + níbé } \\
\text { dé + yébé }\end{array}$} & \multirow{7}{*}{$\begin{array}{l}\text { est prononcé } \\
\text { " } \\
" \\
" \\
" \\
"\end{array}$} & \multirow{2}{*}{$\left.\begin{array}{l}{\left[\begin{array}{ll}- & -\end{array}\right]} \\
{\left[\text { dé }_{1} \text { zú }\right.}\end{array}\right]$} & \multirow{3}{*}{$\begin{array}{l}\text { 'prends une tête' } \\
\text { 'prends dix' }\end{array}$} \\
\hline & & & \\
\hline & & [dé píé $_{2}$ ] & \\
\hline & & {$\left[\right.$ dé $_{1}$ yír 2$]$} & 'prends une maison' \\
\hline & & [dée kúór 2 ] & 'prends une gourde' \\
\hline & & [dé ní $_{2} \mathrm{~b} \varepsilon_{2}$ ] & 'prends des personnes' \\
\hline & & [dé y $_{2}$ bé 2 ] & 'prends un frère' \\
\hline & & & \\
\hline b. nów + zú & est prononcé & [nów zú $_{2}$ ] & 'attrape une tête' \\
\hline nów + píé & & [nów píé$_{2}$ ] & 'attrape di \\
\hline nów + yír & " & [nów yír $\left.{ }_{2}\right]$ & 'attrape une maison' \\
\hline nów + kúór & " & [nów kv́ós $\left._{2}\right]$ & 'attrape une gourde \\
\hline
\end{tabular}

Ainsi, on peut interpréter phonologiquement les bases [dé], [nów] et avec elles, toutes les autres bases du même type telles que [gấ], [GÉl], [fór], [fáá], [vúúl], [cến], comme dans (16).

\begin{tabular}{|c|c|c|c|}
\hline [dé] & /déed/ & $</$ dê $/$ & 'prends' \\
\hline [nów] & < /nóg̀/ & & 'attrape' \\
\hline [gấ] & < /gáǹ/ & & 'couche-toi' \\
\hline [Gél] & $<$ /6źl/ & & 'arrache (feuilles)' \\
\hline [fór] & $<$ /fód/ & & 'sirote' \\
\hline [fáá] & < /fáà/ & & 'retire un objet' \\
\hline [vúúl] & $<$ /vúul/ & & 'passe au feu' \\
\hline [cến] & /cénǹ/ & & 'va/marche' \\
\hline
\end{tabular}

3.2.2 Les tons flottants bas dans les bases nominales. Contrairement aux bases verbales qui apparaissent comme telles dans le lexique, les bases nominales sont toujours associées à un suffixe de classe, et sont donc attestées dans le lexique exclusivement sous forme de constituants nominaux. Pour obtenir donc des bases nominales, il faut réduire chaque constituant à sa forme basique. Pour cela, il faut l'intégrer dans une structure de forme composée, telle que le type de forme structurelle Base nominale + Adjectif qualificatif que l'on trouve dans les syntagmes qualificatifs. Dans ce type de composition, chaque constituant est amené à se dissocier de son suffixe classificatoire pour ne conserver que sa forme de base. Pour éviter des interférences tonales, on fera de sorte que l'adjectif qui accompagne la forme nominale soit à ton bas.

Deux types de constituants à ton haut seront examinés: ceux n'impliquant pas de downstep comme [bíé] 'jeux' dont la structure phonologique /HB/ coincidant avec celle d'une série d'autres constituants est donnée en (11), et ceux impliquant 
un downstep comme [lwé'é] 'poussin' présenté ci-dessous en (17) avec d'autres constituants du même type, tous illustrés sous leurs formes en mots isolés.

\begin{tabular}{|c|c|c|}
\hline & \multicolumn{2}{|c|}{ 7) [lwé'é] } \\
\hline & [fííl'á] & 'petit passage' \\
\hline & [sól'ú] & 'conte' \\
\hline & [cér'é] & 'dieu protecteur de la maison' \\
\hline & [kpówr'á] & 'nom d'arbuste' \\
\hline & [wúl'ú] & 'maladie' \\
\hline
\end{tabular}

Si on réduit à leur forme de base chacun des constituants en (11) on obtient les bases ci-dessous en (18). L'astérisque /*/ signifie que ces bases ne sont jamais utilisées comme telles en mots isolés mais toujours intégrées dans une structure de forme composée. On remarquera que l'adjectif [vlà] au singulier devient [vìc̀l] au pluriel lorsque la base extraite provient d'un constituant au pluriel. Si l'on répète la même opération avec les constituants à downstep, l'on obtient les formes en (19) dans une structure de forme composée.

\begin{tabular}{|c|c|c|c|c|c|}
\hline (18) [bíé] & donne la base & $*\left[b_{1}\right]$ & dans & [bí vì̀̀l] & 'beaux jeux' \\
\hline [léć] & " & *[lá $]^{10}$ & " & [lá vìc̀l] & 'bonnes haches' \\
\hline [níd] & " & $*[$ ní $]$ & $"$ & [ní vlà] & 'bonne personne' \\
\hline [píir] & $"$ & $*[p i ́]$ & $"$ & [pí vìc̀l] & 'bons moutons' \\
\hline [váár] & $"$ & *[vá] & $"$ & [vá vì̀̀l] & 'bonnes feuilles' \\
\hline [yébé] & $"$ & *[yéb] & " & [yé vlà] & 'bon frère' \\
\hline 9) [lwé'é] & donne la base & $*[$ lú $]$ & dans & [lú vlà] & 'beau conte' \\
\hline [fííl'lá] & $"$ & $*[f i ́ t l]$ & $"$ & [fíil vlà] & 'beau passage' \\
\hline [sól'ú] & $"$ & $*$ [sól $]$ & $"$ & [sól vlà] & 'beau conte' \\
\hline [cér'é] & $"$ & *[cér $]$ & $"$ & [cér vlà] & $\begin{array}{l}\text { 'un bon dieu } \\
\text { protecteur' }\end{array}$ \\
\hline [kpówr'a] & $"$ & *[kpówr] & ] & [kpówr vlà] & 'bel arbuste' \\
\hline [wúl'v́] & $"$ & $*[$ wúl $]$ & $"$ & [wúl sũ̀w] & 'grave maladie' \\
\hline [fíwr'á] & $"$ & $*[$ fíwr] & " & [fíwr vlà] & 'beau fouet' \\
\hline
\end{tabular}

A présent, nous sommes parvenus au point recherché de la description où il faut démontrer le nombre de tons phonologiques impliqués dans ces bases nominales. Pour connaître réellement les tons des bases comme [bí] d'un côté et [lú]

10 Dès qu'un constituant comme /láa-غ̇/ > [léć] perd son suffixe /E/ du pluriel, la base reprend la voyelle du constituant de la forme du singulier, ici la voyelle $a$ dans [lár]; mais la forme pluriel sera exprimée par l'adjectif qui lui sera associé. Par contre dans le cas de l'exemple [pír] il y a une alternance de voyelle $\mathrm{t} / \mathrm{i}$ lorsque le mot passe du singulier [pír] au pluriel [pírr]. 
de l'autre, nous allons les faire suivre par des constituants à ton haut comme [dếnw], [bíér] [wów], [túúlé], [kórá], qui sont prononcés comme tels en formes isolées. Si le ton haut de ces constituants est abaissé, cela veut dire que la base qui précède immédiatement a un ton flottant bas caché. Par contre, si le ton haut n'est pas abaissé, cela veut dire que la base qui les précède n'a qu'un ton haut simple. En se rappelant que la base [bí] vient de [bíé] au pluriel et [bír] au singulier, l'adjectif associé à cette base doit être au pluriel, soit respectivement [dến], [bébé], [wówr], [túúlí], [kóró], le pluriel correspondant des adjectifs ci-dessus cités en mots isolés. On obtient alors les réalisations en (20a). On peut donc interpréter les bases de type [bí] comme n'ayant qu'un seul ton phonologique haut, parce qu'il n'y a pas d'abaissement tonal, d'où les exemples en (20b).

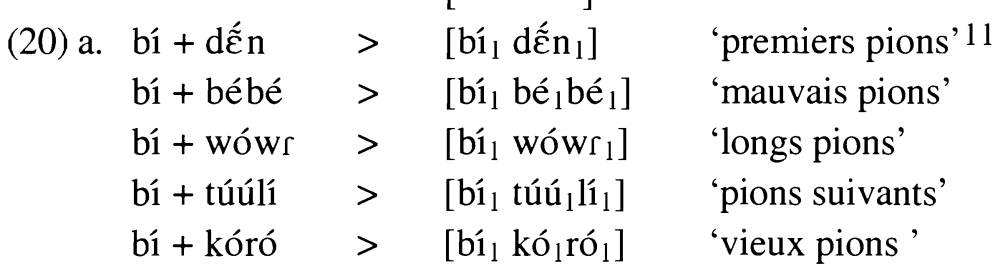

$\begin{aligned} \text { b. } \text { bí] } & </ \text { lbill } & \text { [pí] } & </ \text { /pí/ } \\ \text { lá] } & </ l a ́ / & {[v a ́] } & </ v a ́ / \\ \text { ní] } & </ n i ́ / & {[y \varepsilon ́ b] } & </ y \varepsilon ́ b /\end{aligned}$

Revenons à la comparaison entre [bí] et [lú]. Lorsque l'on remplace la base [bí] par la base [lú] dans le même contexte, la réalisation change. Dans les exemples cités en (21a), les adjectifs sont sous la forme du singulier citée en (20), ce qui ne change absolument rien à la démonstration tonale poursuivie. Pour nous rassurer que cette baisse tonale n'est pas accidentelle, remplaçons la base [lú] par la base [fíil] et reprenons comme deuxième unité tous les mots utilisés au pluriel en (20a). Le ton haut de la deuxième unité est également abaissé en (21b). Face à ce résultat, on déduit que les bases nominales du type de [lú], [fí́l], ont un ton flottant bas caché qui est à l'origine de l'abaissement du ton haut observé en (21c).

\begin{tabular}{|c|c|c|c|}
\hline (21) a. lú + dé́nw & $>$ & {$\left[1 u_{1} \mathrm{~d} \tilde{\varepsilon} \mathrm{w}_{2}\right]$} & 'premier poussin' \\
\hline lú + bíér & $>$ & [lú ${ }_{1}$ bíér 2 ] & 'mauvais poussin' \\
\hline lú + wów & $>$ & [lú wów $_{2}$ ] & 'long poussin' \\
\hline lú + túúlé & $>$ & [lú túú $_{2}$léé$_{2}$ ] & 'poussin suivant' \\
\hline lú + kórá & $>$ & [lú kó $_{2}$ rá $\left.{ }_{2}\right]$ & 'vieux poussin' \\
\hline
\end{tabular}

11 Pour une question de sémantique, le mot 'jeux' est remplacé par le mot 'pions'. 


\begin{tabular}{|c|c|c|c|c|}
\hline \multirow[t]{3}{*}{ (21) b. } & 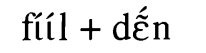 & $>$ & {$\left[\mathrm{fíi}_{1} \mathrm{l}_{1} \mathrm{~d} \tilde{\varepsilon} \mathrm{n}_{2}\right]$} & 'premiers passages' \\
\hline & fúil + bébé & $>$ & [fí́l ${ }_{1}$ bé $_{2} b_{2}$ ] & 'mauvais passages' \\
\hline & fúćl + wówr & $>$ & $\begin{array}{l}{\left[f_{i i ́ l} l_{1} \text { wówr } 2\right.} \\
{\left[\begin{array}{cc}- & -\end{array}\right]}\end{array}$ & 'longs passaages' \\
\hline \multirow[t]{6}{*}{ c. } & lú + zú & $>$ & [lú $\left.\mathbf{u}_{1} z \mathbf{u}_{2}\right]$ & 'tête de poussin' \\
\hline & lú + gbér & $>$ & [lú gbé $\left._{2}\right]$ & 'patte de poussin' \\
\hline & lú + núór & $>$ & [lú 1 núór 2$]$ & 'bouche de poussin' \\
\hline & fí́l + zú & $>$ & {$\left[f_{i ́ c} l_{1} z_{2} u_{2}\right]$} & 'tête de passage' \\
\hline & fííl + gbér & $>$ & [fíúl $\left.\mathrm{gbé}_{2}\right]$ & 'branche de passage' \\
\hline & fúíl + núór & $>$ & [fúíl $l_{1}$ núór $\left.{ }_{2}\right]$ & 'début du passage' \\
\hline
\end{tabular}

On peut remplacer les adjectifs dans la deuxième position et associer aux bases [lú] et [fí́l] des constituants nominaux tels que [zú], [gbér], [núór], pour former des noms composés non figés qui suivent une règle de reproduction syntaxique. Dans cette situation, le ton haut des constituants nominaux subit le même sort que dans les autres cas comme on peut le voir en (21c). Ainsi, qu'il s'agisse de structure syntaxique à base d'adjectif qualificatif $(21 \mathrm{a}, \mathrm{b})$ ou de structure syntaxique à base de nom composé (21c), il y a abaissement du ton haut de la deuxième unité: cela signifie que les bases [lú], [fííl] et, avec elles, toutes les bases du même type, ont chacune un ton flottant bas caché. C'est ce même ton flottant bas caché qui provoque le downstep observé en (17). Nous les interprétons donc phonologiquement comme montré en (22).

\begin{tabular}{|c|c|c|c|}
\hline (22) & [lú] & $<$ & /lú(ù) \\
\hline & [fúíl] & $<$ & /füul/ \\
\hline & [sól] & $<$ & /sól// \\
\hline & [cér] & $<$ & /céd/ \\
\hline & [kpíwr] & $<$ & /kpógd/ \\
\hline & [wól] & $<$ & /wúl// \\
\hline & [fííwr] & $<$ & /füı̀t/ \\
\hline
\end{tabular}

L'autre question à résoudre immédiatement est la suivante: est-ce que tous les constituants nominaux impliquant un downstep maintiennent toujours un ton flottant bas dans la base quand celle-ci se dissocie de son suffixe classificatoire? La réponse, par anticipation, est non. Pour le prouver, voici une autre liste de constituants nominaux impliquant un downstep. Nous allons d'abord dissocier les bases de leurs suffixes en nous servant du même type de structure composée utilisé ci-dessus ; plus loin nous allons prouver qu'elles n'ont qu'un ton haut simple. 


\begin{tabular}{|c|c|c|c|c|c|}
\hline ) $[$ ná & donne la & *[náa] & dans & [náa vlà] & 'une belle vache' \\
\hline$[\mathrm{pr}$ & 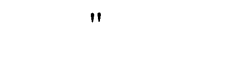 & *[nmấ] & & [nmã́ vlà] & 'une belle \\
\hline [jé'́l] & " & *[jél] & . & [jél & 'un bel oeuf' \\
\hline [vá'á] & $"$ & *[vá] & 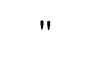 & [vá vlà] & 'une belle feuille' \\
\hline [kó'ŕc] & " & *[kór $]$ & " & [kór vlà] & 'une belle joue' \\
\hline
\end{tabular}

En plaçant un mot à ton haut (un des adjectifs cités ci-dessus, [dếw], par exemple) après ces bases, on s'aperçoit que le ton haut n'est pas abaissé contrairement à ce qui est observé en (21). Pour plus de certitude, remplaçons l'adjectif [dẼ́w] par [biér] 'mauvais' (24b). On constate qu'il n'y a pas d'abaissement tonal. Pour davantage de preuves, on peut aussi, à la places des adjectifs, associer à ces bases des constituants nominaux comme [gấn], [gbér], [péwr], pour former des structures syntaxiques à base de nom composé. On constate qu'il n'y a pas d'abaissement tonal non plus (24c).

\begin{tabular}{|c|c|c|c|c|}
\hline a. & 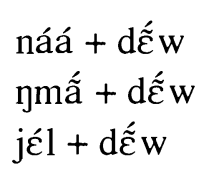 & $\begin{array}{l}> \\
> \\
>\end{array}$ & 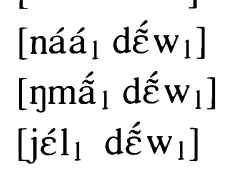 & $\begin{array}{l}\text { 'première vache' } \\
\text { 'première tourterelle' } \\
\text { 'premier oeuf' }\end{array}$ \\
\hline b. & $\begin{array}{l}\text { náá + bíér } \\
\text { jmấ + bíér } \\
\text { jél + bíér }\end{array}$ & $\begin{array}{l}> \\
> \\
>\end{array}$ & 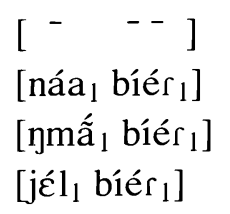 & $\begin{array}{l}\text { 'mauvaise vache' } \\
\text { 'mauvaise tourterelle' } \\
\text { 'mauvais oeuf' }\end{array}$ \\
\hline c. & $\begin{array}{l}\text { náá + gấn } \\
\text { Đmấ + gbér } \\
\text { jél + péwr }\end{array}$ & $\begin{array}{l}> \\
> \\
>\end{array}$ & 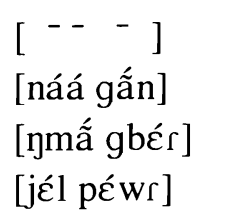 & $\begin{array}{l}\text { 'peau de vache' } \\
\text { 'patte de tourterelle' } \\
\text { 'coque de l'oeuf' }\end{array}$ \\
\hline
\end{tabular}

Ainsi, il apparaît clairement que dans les trois cas montrés en (24), le ton flottant bas a disparu; il n'y a pas d'abaissement tonal du ton haut de l'adjectif ni du constituant nominal qui suit.

Pourquoi le ton flottant bas a-t-il disparu dans ce cas-ci, c'est la question qu'il faut à présent résoudre. Il semble que le downstep lui-même dans ces constituants soit maintenu seulement dans les formes lexicales actuelles de ces constituants: il s'est lexicalisé du fait qu'il n'est plus motivé et apparaît aujourd'hui sous une forme figée. Ceci signifie que l'on ne peut plus, dans ces constituants, séparer le ton flottant bas du ton haut imparti au suffixe de classe. Il ne peut plus non plus se maintenir sur la base, à l'exemple du ton flottant bas identifié dans les bases du type /lû \%. En réalité, la grande différence entre les bases en (21) impliquant un downstep et celles en (24) ci-dessus se situe au niveau lexical. En effet, nous 
montrerons, plus bas, que si le singulier de ces noms en (23) est sous forme de downstep, leur pluriel apparaît plutôt sans downstep (25). Trois arguments expliquent pourquoi les tons flottants bas dans ces noms se comportent de cette façon.

Il y a, d'abord, l'idée avancée d'une réorganisation morphologique du groupe voltaïque [cf. Manessy 1975] aboutissant à la création d'une surpluralisation dans les noms de certaines langues (par exemple, en moore [wááf'ó]/[wíísì] 'serpent(s)', en dagara [wá'áb]/[wíír]), y compris le dagara. Ce phénomène de surpluralisation fait que les nouveaux tons qui se sont sur-ajoutés sont instables et ne peuvent pas se maintenir en l'absence du suffixe et de son ton.

Il y a, ensuite, le problème fondamental de changement de classe des suffixes. Les constituants à downstep, dont il est question, auraient perdu leurs suffixes vocaliques pour ne garder que des consonnes qui appartiennent à la base ou qui sont ré-interprétées comme une partie des bases - ce qui justifie le fait qu'ils soient lexicalisés. On peut, du reste, sur la base du clivage singulier/pluriel se rendre compte que lorsque le ton flottant bas n'est plus motivé comme ton de dérivatif, il disparaît au pluriel et n'entraîne plus de downstep tel qu'indiqué en (25). Ceci n'est pas du tout le cas des autres constituants à downstep donnés en (17). En effet, comme ces constituants n'ont pas été frappés par le processus du changement de suffixe classificatoire, ils ont maintenu le downstep aussi bien au singulier qu'au pluriel (26).

Singulier Pluriel

$\begin{array}{lll}\text { [jmán'ń] } & \text { [Đmámá] } & \text { 'tourterelle(s)' } \\ \text { [nín'ń] } & \text { [nímé] } & \text { 'dent(s)' } \\ \text { [kó'ŕc } & \text { [kóró] } & \text { 'joue(s)' } \\ \text { [jé'́i] } & \text { [jéle] } & \text { 'oeuf(s)' }\end{array}$

(26)

$\begin{array}{ll}\text { [lwé'é] } & \text { [lúl'í] } \\ \text { [fíil'á] } & \text { [fíil'í] } \\ \text { [sól'ú] } & \text { [sól'í] } \\ \text { [cér'é] } & \text { [cér'í] } \\ \text { [kpówr'á] } & \text { [kpáwr'í } \\ \text { [wúl'v́] } & \text { [wól'í] }\end{array}$

'poussin(s)'

'passage(s)'

'conte(s)'

'dieu(x) protecteur(s) de maison(s)'

'nom(s) d'/des arbuste(s)'

'grave(s) maladie(s)'

Enfin, le phénomène de lexicalisation du downstep à la forme exclusive du singulier n'est pas un phénomène propre au dagara, on le retrouve aussi en mooré de Ouagadougou [Kaboré 1985] où tous les suffixes vocaliques de classe sont pourtant attestés. En moore, on pense toutefois que ce phénomène est causé par une sursuffixation des formes du singulier, ce qui explique la disparition du ton supplémentaire au pluriel entraînant ainsi une absence de downstep dans le pluriel de ces noms [cf. Kaboré 1985]. 
(27) Singulier Pluriel

$\begin{array}{lll}\text { [wááf'ó] } & \text { [wíisì] } & \text { 'serpent(s)' } \\ \text { [jéll'é] } & \text { [jéllà] } & \text { 'oeuf(s)' } \\ \text { [nááf'ó] } & \text { [níisì] } & \text { 'boeuf(s)' }\end{array}$

De cette partie de l'analyse, retenons ceci: tout d'abord, parmi les bases verbales, certaines ont des tons flottants bas capables d'abaisser le ton haut qui suit, tandis que d'autres bases, apparemment de même structure canonique que les premières, n'ont qu'un seul ton haut. Il y a, ensuite, le cas des bases nominales qui ont, en plus du ton haut, un ton flottant bas qui provoque un downstep au sein des constituants. Dans ce dernier groupe, il faut encore établir un clivage: il y a les constituants nominaux à downstep (17) qui maintiennent toujours le ton flottant bas dans la base quand ils sont dissociés de leurs suffixes de classe (cf.21), et des constituants à downstep à l'instar de ceux en (23) qui, en se dissociant de leurs suffixes de classe, perdent du coup le ton flottant bas (cf. 24). Cela nous amène à affirmer que le downstep dans ce dernier groupe de constituants s'est lexicalisé et est aujourd'hui figé, de sorte que le ton flottant bas qui l'a déclenché n'est plus isolable du downstep et n'est plus autonome.

Un autre fait général et important à mettre en relief dans cette partie de l'analyse, est la règle du mapping ou recouvrement [Leben 1976]. L'idée générale de cette règle est que l'association des tons à la syllabe se fait durant la formation des unités lexicales; selon Leben, il ne devrait plus rester des tons flottants nonassociés après la formation lexicale. La lecture que nous faisons de cette règle, compte tenu de la structure spécifique et de l'état organisationnel [Hagège 1982] du dagara, est assez nuancée: pour nous, c'est cette règle même qui génère les tons flottants en dagara. En effet, les différents types de tons flottants, qu'ils proviennent des suffixes nominaux ou verbaux, ou des dérivatifs nominaux ou verbaux, sont générés par la règle du mapping. Cette règle est donc redéfinie cidessous pour le besoin du dagara.

1) Quand il y a seulement un ton dans la base, la règle consiste à imposer ce ton de base à tous les morphèmes qui lui sont associés, au détriment de leur(s) propre(s) ton(s) qui devient/deviennent flottant(s).

1.1. Ton de la base du constituant nominal
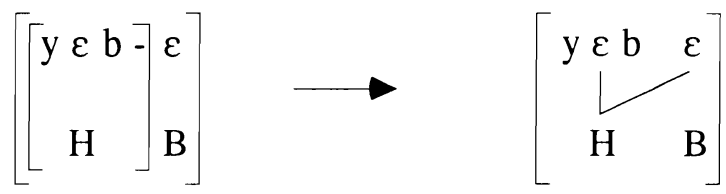

'frère'

Base + Suffixe 
1.2. Ton de la base du constituant verbal

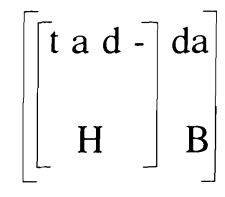

Base + Suffixe
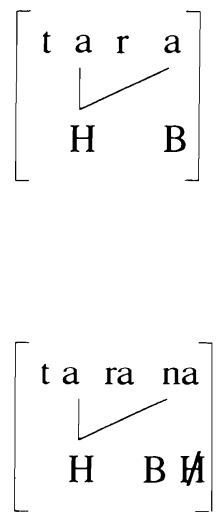

'sois en train d'arrive' 'est en train d'arriver'

1.3. Morphème dérivatif dans la base verbale: /déed/ 'prends'

$\left.\left[\begin{array}{c}\text { d e e } \\ H\end{array}\right] \begin{array}{l}d \\ B\end{array}\right]$

Lexème + dérivatif

$\left[\left[\begin{array}{c}\mathrm{fa} \\ \mathrm{H}\end{array}\right] \mathrm{B}\right]$

Lexème + dérivatif
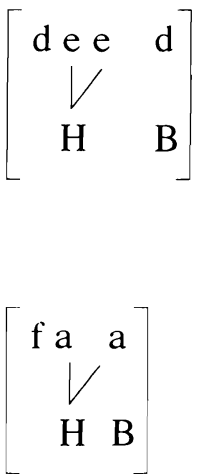

'prends'

'retire un objet'

1.4. Morphème dérivatif dans la base: /fígt-ál 'fouet'

$\left[\left[\begin{array}{c}\mathrm{f} \iota \mathrm{g} \\ \mathrm{H}\end{array}\right] \mathrm{t}\right.$

Lexème + dérivatif

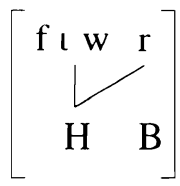

'fouet' 
2) Quand il y a deux tons dans la base tels que dans /gùt-ù/ [gùrú] 'champignon', l'application de la règle consiste à imposer le deuxième ton de la base aux morphèmes suffixaux au détriment de leur(s) propre(s) ton(s) devenu(s) flottant(s).

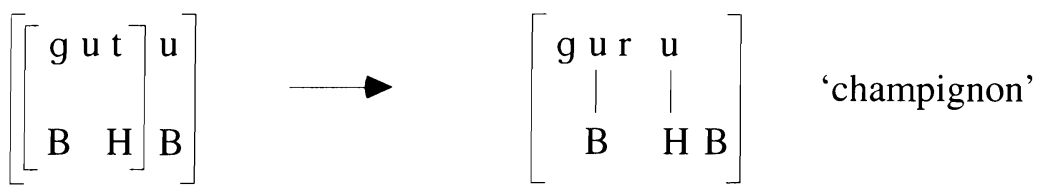

L'association des tons au niveau lexical en dagara est similaire à ce qui a été observé en dschang-bamileke: le lexème, ou du moins la base en ce qui concerne le dagara, a plus de tons que de syllabes. Pulleyblank [1983], dans ce cas de figure, suggère le type suivant d'association qui correspond à ce que nous avons adopté en dagara.

$$
\begin{aligned}
& {\left[\begin{array}{cc}
\mathrm{k} \text { a } \mathrm{n} \\
\mathrm{B} \\
\mathrm{B} & \mathrm{H}
\end{array}\right] \quad \text { 'écureuil' }} \\
& {\left[\begin{array}{c}
g \mathrm{y} \mathrm{a} \\
\mathrm{H} \mathrm{B}
\end{array}\right] \quad \text { 'maison' }}
\end{aligned}
$$

\section{Tout ton flottant bas autorise-t-il la propagation du ton haut précédent?}

Pour répondre à cette question il faut d'abord définir les deux types de tons flottants bas, 'périphériques' et 'internes'.

Le ton flottant bas "périphérique". Ce ton se définit comme ton de suffixe de constituant nominal (11) ou verbal (14b) et/ou de dérivatif des bases verbales (16) en position finale de l'unité qu'il affecte. Il ne s'agit ni d'un ton fictif ajouté pour le besoin de la description, ni d'un ton mobile sans support fixe, mais plutôt d'un ton réel qui a aujourd'hui un support fixe qui n'est pas nécessairement celui qu'il avait originellement. Il est toujours généré par un processus morphologique consistant à inclure son support originel dans la base et à lui re-assigner un nouveau support absolument fixe aujourd'hui (voir Hyman [1976] sur la même question). Il a comme propriété celle d'abaisser le ton haut qui suit, mais surtout (et anticipant la question posée ci-dessus), d'autoriser la propagation du ton haut précédent comme indiqué en (28), (29a,b), (30).

Le ton flottant bas 'interne'. Il se définit essentiellement comme un ton de dérivatif nominal (cf. 22) ou adjectival dans quelques rares cas (cf. 39), qui n'a jamais été re-assigné à un nouveau support, même si le support actuel est aujourd'- 
hui confondu à la base. Comme propriété, ce ton flottant abaisse le ton haut qui suit, comme illustré en (21), et n'autorise pas la propagation du ton haut précédent (cf. 36, 37, 38).

Nous allons répondre à présent à la question ci-dessus, en examinant chaque type de tons flottants bas dans différents cadres syntaxiques (syntagmes, séries verbales, énoncés verbaux, etc.). Pour atteindre le résultat escompté, nous allons prendre d'abord soin de placer en position de X1 des unités sous leurs formes phonologiques, c'est-à-dire, les constituants (nominaux et verbaux) et les bases (verbales et nominales) dont les tons flottants ont été ci-dessus identifiés; nous allons, ensuite, mettre en X2, d'une part, des mots ayant des consonnes transparentes en position initiale, et d'autre part, des mots monosyllabiques à ton bas ou des mots dont la première syllabe—dans des mots à deux syllabes—est à ton bas.

\subsection{Les tons flottants bas "périphériques" autorisent la propagation du ton} haut précédent. On peut affirmer qu'en dagara, tous les tons flottants bas situés à la périphérie d'une unité, abstraction faite de sa catégorie, favorisent la propagation du ton haut précédent sur le mot qui suit. Ainsi, si nous plaçons d'abord les constituants nominaux-/bíè//níd/, /kál/, /lé غ̇/, examinés en (11) et qui sont présentés ici sous leur forme phonologique-en position de X1, et en X2, les constituants verbaux à l'accompli des bases verbales citées en (8a) (dont les formes en mots isolés répondent aux conditions ci-dessus décrites et correspondent respectivement à [pùná], [fàná], [tìná], [sòná], [cìná], [kàná], [kpàná], et enfin [hèlìná] que, pour une raison de structure, nous remplaçons par [hàbá]), on s'aperçoit alors que le ton flottant bas de /níd/ en X1 ne peut pas bloquer la propagation du ton haut précédent. Ci-dessous, en (28), on a mis en relation un nom sujet suivi d'un verbe qui assume la fonction prédicat.

\begin{tabular}{|c|c|c|c|c|}
\hline 8) níd + pùná & /níd pûná/ & $>$ & [ní ${ }_{1}$ pú ${ }_{1}$ n’á $\left._{2}\right]$ & 'quelqu'un a fleuri' \\
\hline níd + fàná & /níd fâná/ & $>$ & [nír f fá $_{1}$ n’á 2$]$ & 'quelqu'un est foutu' \\
\hline níd + tìná & /níd tinál & $>$ & [nír tíl$_{1}$ n’á & 'quelqu'un a vomi' \\
\hline níd + sòná & /níd sôná/ & $>$ & [nír ${ }_{1}$ són $\left.{ }_{1}{ }^{\prime} a_{2}\right]$ & 'quequ'un a épicé' \\
\hline níd + cìná & /níd cîná/ & $>$ & [nír ${ }_{1}$ cí ${ }_{1}$ n’á & 'quelqu'un a parié' \\
\hline níd + kàná & /níd kâná/ & $>$ & [nír 1 ká n’á 2 ] & 'quelqu'un a cassé' \\
\hline níd + kpàná & /níd kpâná/ & $>$ & [ní $\Gamma_{1}$ kpá ${ }_{1}$ ’á 2$]$ & ‘quelqu'un a cloué' \\
\hline níd + hàbá & /níd hábál & $>$ & [nír ${ }_{1}$ há $b^{\prime} a_{2}$ ] & 'quelqu'un a caché' \\
\hline
\end{tabular}

En effet, le ton haut du constituant /níd/ se propage sur la première syllabe de $\mathrm{X} 2$, comme si le ton bas flottant lui-même était totalement absent de /níd/, repoussant ainsi le ton bas initial de la première syllabe de $\mathrm{X} 2$, lequel, à son tour, provoque un downstep en rabaissant le ton haut final en $\mathrm{X} 2$.

Prenons un deuxième exemple semblable syntaxiquement aux exemples en (28) mais différent par rapport à la structure du nom qui assume la fonction sujet, le 
constituant [yébé] pour /yébè/ 'frère' qui a déjà été analysé en (11) et dans lequel, rappelons-le, le ton bas sur le /-bè/ est flottant (cf. 10c).

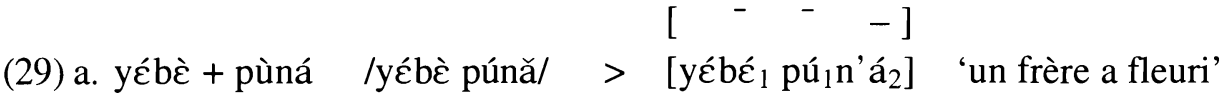

$$
\begin{aligned}
& \text { yébè + fùná /yébè fúnă/ > [yébé } \text { fúl }_{1}{ }^{n}{ }_{2} \text { ] 'un frère a cuit } 2 \text { fois' } \\
& \text { yébè + tìná /yébè tínă/ > [yébé } \text { tíl }_{1} n^{\prime}{ }_{2} \text { ] 'un frère a vomi' } \\
& \text { yébè + sòná /yébè sónă/ > [yébé } \left.{ }_{1} \text { só }_{1} n^{n} \text { á }_{2}\right] \text { 'un frère a épicé' } \\
& \text { yébè + cìná /yébè cínă/ > [yébé }{ }_{1} \text { cí }{ }_{1}{ }^{\prime} \text { 'á }{ }_{2} \text { ] 'un frère a parié' } \\
& \text { yébè + kàná /yébè kánă/ > [yébé }{ }_{1} \text { ká }{ }_{1} n^{\prime} a_{2} \text { ] 'un frère a cassé' } \\
& \text { yébè + kpàná /yébè kpánă/ > [yébé } \text { kpá }_{1} \text { n'á }_{2} \text { ] 'un frère a cloué' }
\end{aligned}
$$

Le ton flottant bas dans /yébè/ se comporte exactement comme dans le cas de /níd/, et autorise la propagation du ton haut de /yé-/ sur la première syllabe du mot qui suit, entraînant, tout comme dans les exemples précédents en (28), un downstep sur la dernière syllabe de X2.

\begin{tabular}{|c|c|c|c|c|}
\hline \multirow{5}{*}{ (29) b } & \multirow{5}{*}{$\begin{array}{l}\text { b. yébè + pì̀ } \\
\text { yébè + tàbà } \\
\text { yébè + kù̃̂̃ } \\
\text { yébè + sù̃̂́ } \\
\text { yébè + mà }\end{array}$} & /yع́t & 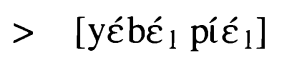 & 'panier du frère' \\
\hline & & /yćbè tábà/ & [yébé tábá $_{1}$ ] & 'tabac du frère' \\
\hline & & /yébè kúoǹ/ & [yébé kúv́̃ $_{1}$ ] & 'eau du frère' \\
\hline & & /yćbè súoñ/ & 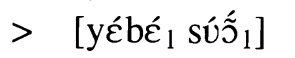 & 'ellerie du frère' \\
\hline & & /yébè mâ / & > [yćbé 1 má $\left.{ }_{1}\right]$ & 'mère du frère' \\
\hline
\end{tabular}

Du point de vue syntaxique, si l'on combine X1 (/yébè/) avec le X2 formé de constituants nominaux dont les formes isolées sont respectivement [pì̀], [tàbà], [kùố], [sì̃̂], [mà], pour créer des syntagmes complétifs, on constate que la propagation du ton haut se déroule aussi de la même façon dans ce cadre syntaxique. En voici en (29b) une illustration.

A présent, examinons la réaction du ton flottant dans les bases verbales, celles étudiées notamment en (16) et ayant respectivement comme structure phonologique les formes /dé /, [nóğ/, /gáñ/, /bél/. Que se passe-t-il lorsque X1 est l'une de ces bases verbales, et $\mathrm{X} 2$, chacune des bases verbales employées comme deuxièmes éléments en (8a) et qui ont toutes en position initiale une consonne transparente? L'illustration en (30) est donc faite de séries verbales du type propre aux langues voltaiques [Manessy 1985] tout comme en (8a). Les faits demeurent les mêmes et le ton flottant bas de la base verbale autorise la propagation du ton haut précédent. Dans ce cas-ci, le ton bas initial de X2, délogé de son support, devient un ton flottant bas au niveau phonologique. 


\begin{tabular}{|c|c|c|c|c|}
\hline (30) dé + pù & /dê pû / & $>$ & [dé pú] & 'prends et fleuris' \\
\hline dé + fà & /dê fâ / & $>$ & [dé fá] & 'prends et sois foutu' \\
\hline dé + tì & /dê tîl/ & $>$ & [dé tí] & 'prends et vomis' \\
\hline dé + sò & /dêsô/ & $>$ & [dé só] & 'prends et épice' \\
\hline dé + cì & /dêcîl & $>$ & [dé cí] & 'prends et parie' \\
\hline dé + kà & /dê kâ / & $>$ & [dé ká] & 'prends et casse' \\
\hline dé + kpà & /dê kpâ / & $>$ & [dé kpá] & 'prends et cloue' \\
\hline dé + hàb & /dê háb̆/ & $>$ & [dé háb] & 'prends et coince' \\
\hline
\end{tabular}

Lorsque les constituants verbaux à la forme injonctive progressive tels que /táddà/, /kóodò/, /kúldò/ (cf. 14b), assument la fonction prédicat, cela ne change rien à l'universalité de la règle. En effet, lorsque ceux-ci sont situés en X1, et qu'en $\mathrm{X} 2$ on place les mêmes constituants qu'en (29b), le ton haut de /táddà/ se propage sur le mot suivant ou la première syllabe du mot suivant. On observe, en effet, que malgré la présence du ton flottant bas final de /táddà/, le ton haut de ce dernier se propage sur $\mathrm{X} 2$ dont le ton bas initial délogé devient, à son tour, un autre ton flottant.

\begin{tabular}{|c|c|c|c|c|}
\hline (31) táddà + pì ह̀ & /táddà píč/ & $>$ & [tárá pí ć] & $\begin{array}{l}\text { 'sois en train de t'approcher } \\
\text { du panier' }\end{array}$ \\
\hline táddà + tàbà & /táddà tábà/ & $>$ & [tárá tábà] & $\begin{array}{l}\text { 'sois en train de t'approcher } \\
\text { du tabac' }\end{array}$ \\
\hline táddà + kù̃̄ & /táddà kúoǹ/ & $>$ & [tárá kúó] & $\begin{array}{l}\text { 'sois en train de t'approcher } \\
\text { de l'eau' }\end{array}$ \\
\hline táddà + sù̃̃ & /táddà súsǹ/ & $>$ & [tárá súố] & $\begin{array}{l}\text { 'sois en train de t'approcher } \\
\text { de la sorcellerie' }\end{array}$ \\
\hline táddà + mà & /táddà má / & $>$ & [tárá má] & $\begin{array}{l}\text { 'sois en train de t'approcher } \\
\text { de la mère' }\end{array}$ \\
\hline
\end{tabular}

Ainsi, comme point central de l'analyse nous pouvons affirmer ceci : dans tous les exemples que nous venons d'examiner, un des points extrêmement importants est que tout se passe comme si le ton flottant bas dans X1 était inexistant. De ce fait, si l'on maintient en X2, par exemple, les mêmes verbes qu'en (8a), et que l'on place dans la position de X1 un mot n'ayant qu'un ton haut simple sans ton flottant bas, le morphème [tá] dans [tá...V] marquant la négation en dagara, on obtient le même résultat qu'avec la présence du ton flottant bas dans /níd/, /yébè/, /dé /, /táddà/. Voici une illustration dans laquelle l'abaissement du ton haut du segment [...V́] de la négation est provoqué par le ton bas de X2 qui a été délogé de son support par le ton haut de la négation [tá]. L'intérêt du point de vue syntaxique est de montrer que lorsque $\mathrm{X} 1 \mathrm{n}$ 'a qu'un ton haut simple (sans ton flottant) on obtient le même résultat qu'avec la présence du ton flottant. 


\begin{tabular}{|c|c|c|c|c|c|}
\hline \multirow[t]{7}{*}{$(32) \mathrm{a}}$. & tá + pù é & /tá pú é/ & $>$ & [tá pú'é ] & 'ne fleuris pas' \\
\hline & tá + fù é & /tá fû̀ é/ & $>$ & [tá fú'é] & 'ne cuis pas deux fois' \\
\hline & tá + tì é & /tá tî̀ é/ & $>$ & [tá tí’é] & 'ne vomis pas' \\
\hline & tá + sò ع́ & /tá sô ع́l & $>$ & [tá só'ć] & 'n'épice pas' \\
\hline & tá + cì ع́ & /tá cî̀ ع́l & $>$ & [tá cí' é] & 'ne parie pas' \\
\hline & tá + ká ع́ & /tá kâ é/ & $>$ & [tá ká'é] & 'ne casse pas' \\
\hline & tá + kpá ć & /tá kpá é/ & $>$ & [tá kpá'é] & 'ne cloue pas' \\
\hline \multirow[t]{6}{*}{ b. } & ní + plà & /ní pílà/ & $>$ & [ní plá] & 'une personne blanche' \\
\hline & 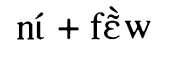 & /ní féngф/ & $>$ & [ní fर̃́w] & 'une personne borgne' \\
\hline & ní + tòr & /ní tódø/ & $>$ & [ní tór] & 'une personne vraie' \\
\hline & ní + sĩ̀w & /ní súngø// & $>$ & [ní sṹw] & 'une personne importante' \\
\hline & ní + còr & /ní códф/ & $>$ & [ní cór] & 'une personne immature' \\
\hline & ní + kồw & /ní kóng ф/ & $>$ & [ní kốw] & 'une personne maigre' \\
\hline
\end{tabular}

Le résultat en (32a) n'est pas unique; car, si à présent on remplace la négation [tá] en X1 par la base /ní/ à un seul ton haut tel que démontré en (20b) et que l'on place en X2 les adjectifs [plà], [fẼ̀w], [tòr], [sũ̀w], [còr], [kồw], qui se présentent comme tels quand ils sont en mots isolés, on obtient le même résultat qu'en (32a): le ton haut de /ní/ se propage sur les adjectifs (32b).

En résumé, retenons que les tons flottants bas des constituants nominaux et verbaux, et ceux des bases verbales, tous qualifiés de "ton flottants bas périphériques", ne peuvent pas bloquer la propagation du ton haut précédent sur X2. Ces tons flottants bas se comportent comme s'ils étaient totalement absents; et le fait qu'ils ont un comportement différent mis en relief dans cette partie de l'analyse soulève un problème autour de la dichotomie saussurienne synchronie/diachronie. On peut alors poser que dans leur mouvance, les tons flottants bas du dagara sont à la croisée de cette dichotomie et que les faits ne sont pas aussi tranchés que le supposait Saussure [1978] lui-même. En dagara, synchronie et diachronie cohabitent, et cela, surtout dans le cas précis des tons; lorsque le ton flottant bas abaisse le ton haut suivant comme en (10), (13), (15a, b), ce ton bas relève de la diachronie, et son effet de la synchronie; tandis que l'incapacité de ces tons flottants bas de bloquer le ton haut précédent est un fait synchronique avec cependant une explication d'ordre diachronique, tout comme on l'observe en Kikuyu [Clements 1979]. En tout état de cause, on est amené à postuler en ce qui concerne l'inertie contextuelle des tons flottants bas que ceux-ci sont appelés à disparaître à court ou à long terme.

Un autre point important concernant la propagation du ton haut des constituants (nominaux et verbaux) et des bases verbales en X1 sur X2, est que, lorsque le ton bas de X2 est délogé par la propagation du haut précédent, il est soumis à trois possibilités de réalisation: 
i) Il se réalise sur une partie du support segmental de $\mathrm{X} 2$, lorsque celui-ci est dissyllabique. En effet, si l'on conserve /táddà/ en X1, et en X2 des constituants nominaux comme [tàbà], [kpòlò], [cغ̀lè], on obtient les réalisations en (33).

\section{(33) táddà + tàbà /táddà tábà/ > [tárá tábà] 'approche-toi du tabac' táddà + kpòlò /táddà kpólò/ > [tárá kpólò] 'approche-toi des termites' táddà + cèlè /táddà célè/ > [tárá célè] 'approche-toi des cris'}

ii) Il provoque aussi un downstep lorsque la situation tonale le permet. C'est ce que nous avons observé dans les exemples en (28), (29a), (32a).

iii) Enfin, lorsqu'il manque de support sur lequel il peut se réaliser, il demeure dans le système comme ton flottant, et dans ce cas, il n'est pas éliminé comme en wobe [Paradis 1984]; il peut abaisser tout ton haut qui suit. Ainsi, si nous prenons les résultats des combinaisons en (30) et que nous associons à l'ensemble de la série verbale ainsi formée, la base verbale [né] 'vois' à ton haut, on obtient les réalisations suivantes dans lesquelles le ton haut de [né] est abaissé (34). Cet abaissement est la preuve que les mots [pú], [fá], [tí], [só], en recevant le ton haut précédent sont devenus phonologiquement des mots à deux tons, d'où l'interprétation /pû /, /fấ/, /tî/, /sôl.

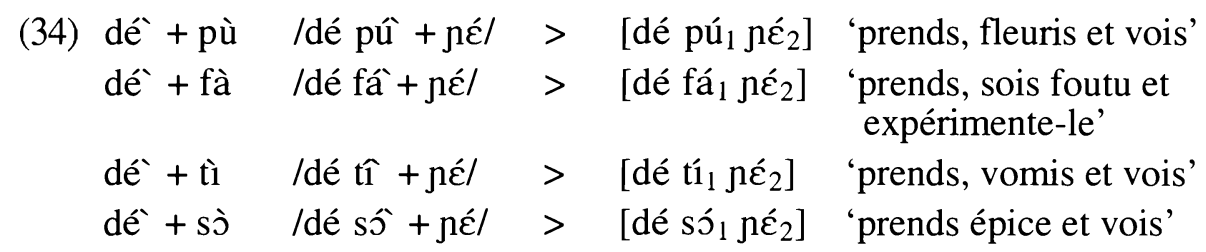

Avant d'examiner les tons flottants bas internes, ouvrons ici un débat théorique sur l'incapacité du ton flottant bas périphérique à bloquer le ton haut précédent. Si nous prenons en compte la sous-règle du well-formedness condition, précisement le point $\mathrm{c}$ de la règle, qui stipule que les traits d'association ne doivent pas se croiser, on se butte à un problème. En effet, si l'on veut éviter que les traits se croisent, il faut d'abord, postuler une règle "d'alimentation" (feeding rule) dont l'application aura pour effet d'éliminer le ton flottant bas. A la suite de l'application de cette règle éliminant le ton flottant bas en $\mathrm{X} 1$, on peut, seulement à ce moment, appliquer la règle d'association des traits. Pour ce qui concerne la règle d'alimentation elle-même, elle peut-être stipulée comme suit:

(35) Le ton flottant bas est éliminé du système lorsqu'il est suivi par un autre ton bas réalisé tel qu'en (28), (29a,b), (30), (31). Ainsi la règle est:

$$
\text { Ton flottant } \mathrm{B} \rightarrow \varnothing / \ldots \text { Ton } \mathrm{B} \text { réalisé. }
$$

Cette analyse montre que, dans certaines langues comme le dagara, des règles tonales doivent être prises en compte avant l'application de la règle du "Well- 
Formedness Condition". C'est bien là un autre argument qui corrobore l'idée qu'en dagara le "Well-Formedness Condition" n'est pas à considérer comme une "condition", mais plutôt comme une forme canonique qui opère effectivement comme telle dans un certain nombre de langues (voir aussi Rialland [1988] au sujet de la même règle). La règle ci-dessus nous autorise à postuler la représentation cidessous dans laquelle les tons bas flottants sont éliminés lorsqu'ils sont immédiatement suivis par un ton bas réalisé tel que dans l'expression /níd pàgná/ 'quelqu'un a fermé'.

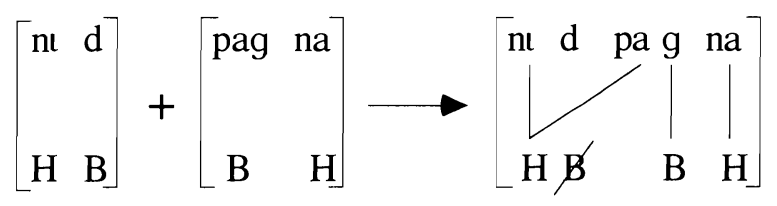

\subsection{Les tons "flottants bas internes" bloquent la propagation du ton haut} précédent. Examinons à présent les tons flottants bas internes illustrés dans des bases nominales impliquant un downstep à l'exemple de /lú'/, /f́́ıl/, /sól/, /céd/, /kpógd/. Le point central est que les tons flottants bas de ces types de base n'autorisent pas la propagation du ton haut qui précède. Avant de procéder à l'illustration, il convient de souligner que, du point de vue syntaxique, ces bases sont soumises à des contraintes telles qu'elles ne peuvent se combiner qu'avec soit des adjectifs pour former des syntagmes qualificatifs, soit des noms pour former des syntagmes complétifs, l'un et l'autre type de syntagme étant inscrits dans la forme structurelle du mot composé. En dehors de ces deux cas, il n'est guère possible d'impliquer d'autres unités syntaxiques telles que, par exemple, la combinaison de /lû́ + constituant verbal du genre */lû́ wàadánà/. Nous limiterons donc nos illustrations à ces deux types d'unités syntaxiques.

Venons-en aux exemples: associons à la base /lû́ / en X1, des adjectifs qualificatifs en $\mathrm{X} 2$ ayant à la fois un ton bas simple, des consonnes transparentes en position initiale dans des unités comme [plà], [fềw], [tòr], [sũ̀w], [còr], [kồw], déjà utilisées (32b) ; il s'agit en fait d'adjectifs qualificatifs dont on a la preuve que les tons bas sont aussi sujets à l'influence du ton haut précédent tel que démontré en (32b); on observe avec intérêt le résultat en (36).
(36) lû́ + plà /lû́ + pì lá/
[lú plà] 'poussin blanc'

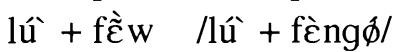
[lú fềw]
'poussin borgne'

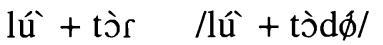
[lú tòr]
'poussin effectif'
lû̀ + sũ̀ /lû́ + sùng $\phi /$
[lú sũ̀w]
'poussin grand'
lû́ + còr $/ /$ î + còd $\phi /$
[lú còr]
'poussin immature'
lû́ + kồw /lû + kòng
[lú kồw]
'poussin maigre' 
Ainsi, on remarque que malgré la présence des consonnes transparentes en position initiale de $\mathrm{X} 2$ - qui favorisent habituellement la propagation du ton haut précédent-le ton flottant bas de /lú / bloque la propagation du ton haut précédent et empêche ce dernier d'étendre son domaine jusqu'au deuxième mot, c'est-à-dire, juqu'à X2. Pour avoir la certitude que ce résultat ne constitue pas un fait isolé, remplaçons la base /lú'/par la base [cér] </céd/ qui vient de [cér'é] 'dieu protecteur' et maintenons les mêmes adjectifs utilisés en X2 en (36).

$$
\begin{aligned}
& \text { céd + plà /céd + pì lá/ [cér plà] 'un dieu protecteur blanc' }
\end{aligned}
$$

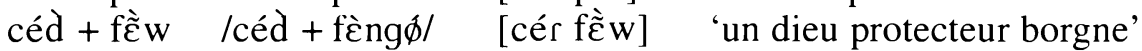

$$
\begin{aligned}
& \text { céd }+ \text { tòr } / \text { céd }+ \text { tòd } \phi / \text { [cér tòr] 'un dieu protecteur authentique' } \\
& \text { céd }+ \text { sũ̃w /céd }+ \text { sùng } \phi / \text { [cér sĩ̃w] 'un dieu protecteur important' } \\
& \text { céd }+ \text { còr /céd }+ \text { còd } \phi / \text { [cér còr] 'un dieu protecteur immature' } \\
& \text { céd + kồw /céd + kòngǿ/ [cér kồw] 'un dieu protecteur maigre' }
\end{aligned}
$$

Les faits sont identiques à ce qui est observé en (36): le ton bas de /céd/ empêche la propagation du ton haut précédent. Ce résultat, dans les deux exemples, constitue une exception bien marquée par rapport à la règle générale du fonctionnement du ton flottant bas périphérique examiné en 4.1.

A ce stade de l'analyse, il faut préciser que c'est vraiment le ton flottant bas des bases /lû́/ et /céd/ qui empêche la propagation du ton haut précédent. On a déjà montré (cf. 32b) et on montrera encore plus loin qu'en l'absence de ce ton bas, le ton des mêmes adjectifs peut être délogé au profit du ton haut précédent.

De plus si l'on maintient en X1, les bases /lû́/, /céd/, et que l'on remplace en $\mathrm{X}_{2}$ les adjectifs par des constituants nominaux tels que [pì̀], [mà], [fầw], [tòwr],

\begin{tabular}{|c|c|c|c|c|}
\hline (38) lû́ + pìc̀ & /lû́ + pìc/ & $>$ & [lú pì̀̀] & 'panier à poussin' \\
\hline lû́ + mà & /lû́ + mà & $>$ & [lú mà] & 'mère de poussin' \\
\hline lú + fã̀w & /lû́ + fàngǿ/ & $>$ & [lu fầw] & 'force de poussin' \\
\hline lû́ + tòwr & /lû́ + tòbd $\varnothing /$ & $>$ & [lú tòwr] & 'oreille de poussin' \\
\hline lû́ + sàã̀ & /lû́ + sàanø/ & $>$ & [lú sàà] & 'père de poussin' \\
\hline lû́ + cèl & /lû́ + cغ̀lф̆/ & $>$ & [lú cèl] & 'cri de poussin' \\
\hline lû́ + kùr & /lû́ + kùdø/ & $>$ & [lú kùr] & 'dos de poussin' \\
\hline
\end{tabular}
[sầà], [cèl], [kùr], pour former des noms composés productifs, donc non figés, on s'aperçoit que le ton flottant bas bloque toujours la propagation du ton haut précédent, comme illustré en (38) ci-dessous.

Comme on le voit, le facteur déterminant dans le blocage de la propagation du ton haut n'est pas de nature syntaxique (tenant à la nature du cadre relationnel des deux termes en présence), mais plutôt de nature lexicale (tenant à la double identité phonologique et morphologique du terme mis en jeu; voir Kiparsky [1982, 1985]. A cet égard, nous avons aussi identifié quelques rares cas d'adjectifs que voici, et dont le ton bas, celui de la base /páal/, par exemple, produit les mêmes effets que 
(39) [páál'á] a pour base /páal/ dans

$\begin{array}{llll}\text { [sáwl'a] } & " & / \text { sábl/ } & \\ \text { [píil'a] } & " & \text { /píl/ }\end{array}$

[ - - ]

[páál $l_{1}$ wów $_{2}$ ] 'neuf et long'

[sáwl wów $_{2}$ ] 'noir et long'

[pííl ${ }_{1}$ wów $w_{2}$ ] 'immature et long'

celui de /lû́/, (cf. 36), ce qui confirme bien que le blocage de la propagation du ton haut est d'ordre lexical (cf. aussi (40)) d'abord, parce qu'aucune base verbale ne remplit les conditions tonales pour occuper la position de $\mathrm{X} 1$; ensuite, parce que le ton flottant bas interne relève du lexique par son opposition aux autres tons flottants bas, les tons flottants bas périphériques en l'occurrence.

Pour confirmer notre analyse, examinons d'autres exemples, ici une association de trois unités: la première unité /náa/ est une base nominale analysée en (24a, b, c) comme n'ayant qu'un seul ton haut phonologique. La deuxième unité, le X1, est l'adjectif /páal/ ci-dessus présenté; et la troisième unité, le X2, est représenté par des adjectifs déjà utilisés en (36) et (37). Des trois unités alignées, seules les deux dernières /páal + adjectif/ illustrent ce que nous voulons prouver. Car la présence de /náa/ permet tout simplement d'asseoir un syntagme qualificatif du type /náa páal/ qui se présente comme un seul mot, un mot composé; et comme /náa/ n'a qu'un ton haut simple, il n'a pas d'impact sur le ton haut de /páal/ qui suit, et donc ne modifie en rien les données; au plus, il permet d'asseoir une structure sémantiquement acceptable pour les dagara. Ce qui importe dans ces exemples, c'est simplement la mise en relation du schème tonal de /páal/ et de celui des adjectifs qui suivent. Dans la présentation en (40), la première colonne d'unités est présentée sous forme phonologique. On s'aperçoit que le ton bas flottant de /páal/ bloque la propagation du ton haut précédent, et l'empêche de s'étendre jusqu'au domaine du dernier mot, ici les adjectifs [plà], [f ह்̃w]. C'est pourquoi nous affirmons que ce qui importe, c'est le statut phonologique et morphologique des termes en présence: lorsque cette condition est remplie, le blocage tonal s'applique dans toute sa rigueur.

(40) $\mathrm{X}_{1}+\mathrm{X}_{2}$

\begin{tabular}{|c|c|c|c|}
\hline ó & $>$ & [náá páál plà] & 'une vache ne \\
\hline$\phi \phi /$ & $>$ & [náá páál fẽ̃̀] & ive borgne' \\
\hline & $>$ & [náá páál tòr] & ́ritable' \\
\hline & $>$ & [náá páál sũ̀w] & ourde' \\
\hline & $>$ & [náá páál kồw] & \\
\hline
\end{tabular}

A cette étape de la démonstration revenons encore à l'idée que le blocage du ton haut dans les exemples ci-dessus présentés en (36), (37), (38) est nécessairement occasionné par le ton flottant bas. Remplaçons la base nominale /céd/, lú / par la base /náa/ et gardons toujours les mêmes adjectifs en X2. On constate alors que le ton haut de /náa/ se propage automatiquement et s'étend sur le domaine de 
$\mathrm{X}$ 2, une propagation d'autant bien compréhensible que les consonnes initiales en $\mathrm{X} 2$ sont toutes de nature transparente. En voici en (41) une illustration.

(41)

\begin{tabular}{|c|c|c|c|c|}
\hline náa + plà & $/ \mathrm{n}$ & $>$ & {$[\mathrm{r}$} & che' \\
\hline ná & /náa féng ळ/ & $>$ & [náa f f̃́w] & 'une vache borgne' \\
\hline & /ná & $>$ & [ná & lentique' \\
\hline & /náa & $>$ & [ná & sse' \\
\hline & /náa códø/ & $>$ & [náa & 'une vache immature' \\
\hline
\end{tabular}

Procédons à présent à une autre opération légèrement différente de la précédente: tout en conservant /náa/ en X1 comme segment à ton haut unique, remplaçons les consonnes transparentes en $\mathrm{X} 2$ par des consonnes opaques dans des mots dont les formes isolées correspondent respectivement à: [brè], [vòr], [dồ], [zàw], [jèl]. On a alors les réalisations en (42).

\section{(42)}

\begin{tabular}{|c|c|c|c|c|}
\hline náa + brè & /náa bìrع/ & $>$ & [náá brè] & 'mamelle de vache' \\
\hline náa + vòr & /náa vòt $\varnothing /$ & $>$ & [náá vòr] & 'passages de vache' \\
\hline náa $+\mathrm{d} \tilde{\tilde{\Lambda}}$ & /náa dònǿ/ & $>$ & [náá dõ̀] & 'inimitié de vache' \\
\hline náa + zàw & /náa zàg & $>$ & [náá zàw] & 'étable de vache' \\
\hline náa $+\mathrm{j}$ c̀l & /náa jèlól & $>$ & [náá jèl] & 'partie de viande de vache" \\
\hline
\end{tabular}

Dans cette illustration, on voit effectivement que les consonnes opaques ont fait barrage à la propagation du haut précédent. Il y a donc lieu de souligner ici que si les tons flottants bas internes bloquent la propagation du haut précédent, les consonnes opaques le font également. Cette situation nous conduit à la dernière question de notre analyse, celle du rapport entre les tons flottants bas et les consonnes opaques.

4.3. Ton flottant bas et consonnes opaques: quel rapport? La question que nous devons clarifier à présent est de savoir s'il existe un lien entre les tons flottants bas internes tels que ceux dans les bases comme /lú / 'poussin', /céd/ 'dieu protecteur de la maison', /fiil/ 'passage des animaux', qui bloquent la propagation du ton haut précédent $(36,37,38)$ et les consonnes opaques qui, elles aussi, bloquent la propagation du ton haut précédent (8b), (42).

Deux hypothèses de travail peuvent aider à expliquer la différence de comportement tonal.

La première hypothèse est que les bases comme /lú /, /céd/, /fùı/, ont perdu une consonne opaque qui, dans ce cas, a pu être /b, d, g/, les seules consonnes opaques susceptibles d'apparaître à la fois en position médiane et finale, et que dans cette situation de perte consonantique, les tons flottants bas de ces consonnes ont pu être maintenus dans le système. L'illustration suivante est une tentative de reconstruction de ces bases. L'étoile qui les affecte signifie qu'il s'agit de bases hypothétiques. 
(43)

$$
\begin{aligned}
& \text { *lúb, *lúd, *lúg } \quad \rightarrow \quad * / \text { lû / } \\
& * \text { cédb, *cédd, *cédg } \rightarrow \quad * / \text { céd/ } \\
& \text { *fúılb, *fúıld, *fíılg } \rightarrow \quad * / \text { füıl/ }
\end{aligned}
$$

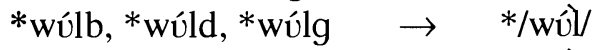

$$
\begin{aligned}
& \text { *fígtb, *fĭgtd, *fïgtg } \rightarrow \quad * / \text { fïgit/ }
\end{aligned}
$$

Toutefois, cette hypothèse est rejetée pour les raisons suivantes:

a) Le ton bas consonantique associé aux consonnes opaques-à l'instar de beaucoup de langues africaines comme le nupe [Hyman 1973:163], le gen [BoleRichard 1983:111] —est seulement actif quand ces consonnes sont en position initiale telle qu'obserbée en (8b), ce qui n'est pas le cas des tons flottants bas dans /lû́/, /céd/, qui apparaissent en position finale et non en position initiale.

b) Il y a des exemples qui prouvent que, lorsqu'une consonne opaque apparaît en position finale, elle s'affaiblit et n'est plus capable d'empêcher la propagation du ton haut précédent sur le mot suivant tel qu'illustré en (44). Si tel est le cas, l'élision de ces consonnes du système n'aurait pas pu générer un ton flottant bas. Les exem-ples en (44) formés de l'association de bases verbales [séb], [yér], [nów] à [tìr] montrent en effet que les consonnes opaques en position finale ne peuvent pas bloquer la propagation du ton haut précédent.

$$
\begin{aligned}
& \text { (44) sćb + tìr /sćb tít/ > [séb tír } \left.\mathrm{r}_{1}\right] \text { 'écris et donne' } \\
& \text { yé } \left.+ \text { tìr /yéd tít/ > [yér } r_{1} \text { tír } r_{1}\right] \text { 'étale et donne' } \\
& \text { nów + tìr /nów tít/ > [nów tír } \left.r_{1}\right] \text { 'prends et donne' }
\end{aligned}
$$

c) Enfin, tout comme en position finale, les trois consonnes /b, g, d/ sont également affaiblies lorsqu'elles sont en position médiane et, par conséquent, ne sont plus capables de bloquer le ton haut qui précède tel qu'illustré en (45) où X1 est représenté par [yéwr] pour /yébtð̋/, [kpégé] pour/kpégè/, [sárá] pour/sádà/, et $\mathrm{X} 2$ par le constituant verbal [pìná] à l'aspect accompli. Cela conduit à la conclusion que leur élimination du système n'aurait pas pu générer des tons flottants. Malgré le fait que les mots en X1 ont chacun, en plus des consonnes opaques, un ton bas final, on peut voir que cela n'empêche pas la propagation du ton haut précédent sur le mot suivant. Cela signifie que dans cette position, les consonnes /b, d, g/ sont affaiblies, et comme telles, ne peuvent pas bloquer la propagation du ton haut précédent.

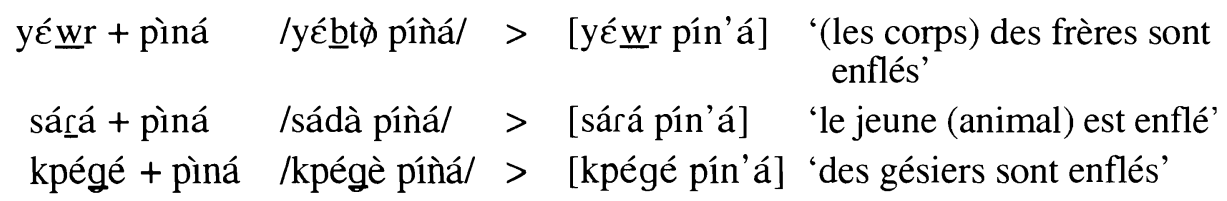


Quant à la deuxième hypothèse, elle stipule que le ton flottant bas dans les mots comme /lú/, /céd/, /fúıl/ est toujours motivé en tant que ton de morphème de dérivatif, morphème qui se définit comme ci-dessus indiqué, c'est-à-dire tout élément, vocalique ou consonantique, qui s'intègre dans un lexème pour former une base avec lui. Cette hypothèse explique la différence entre les deux types de tons flottants. La position "géographique" interne du ton flottant bas dans les basescomme /lú / - les a protégées et empêchées de suivre la vague générale de la remorphologisation et du changement de domaine dans le gur en général, en dagara en particulier. Comme preuve de stabilité de ce ton, on peut avancer deux faits: contrairement aux autres tons flottants bas externes qui disparaissent du système quand le suffixe est dissocié de la base (cf. 24a, b), ce ton flottant bas, dans le même contexte, reste attaché à la base (22). Il est aussi attesté au singulier et au pluriel (26) des constituants qu'il affecte, contrairement à l'autre type de ton flottant bas qui disparaît complètement quand les noms qu'il marque passent du singulier au pluriel (cf. 25).

Cette deuxième hypothèse est par ailleurs justifiée par le fait que les suffixes de classe et leurs tons respectifs sont toujours attestés et servent comme une carapace à la frontière des mots pour protéger les tons internes et les empêcher d'être délogés de leur position et de se ré-analyser comme tons associés à de nouveaux segments. En (46), nous mettons en relief le fait que les suffixes sont vraiment attestés, contrairement aux constituants en (23) dans lesquels les suffixes ne sont pas toujours attestés.

\begin{tabular}{|c|c|c|c|}
\hline (46) [lwé'é] & vient de & /lúù-è/ & 'poussin' \\
\hline [fííl'á] & 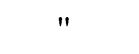 & /fúıl-á/ & 'passage d'animaux' \\
\hline [céd'é] & " & /céd-é/ & 'dieu protecteur' \\
\hline [sól'ú] & $"$ & /sól-ú/ & 'conte' \\
\hline [kpówr’á] & " & /kpígd-á/ & 'nom d'arbuste' \\
\hline [wúl'v́] & $"$ & /wúl-ú/ & 'maladie' \\
\hline
\end{tabular}

L'implication directe de cette hypothèse qui considère que le ton flottant bas dans les bases /lú /, /céd/, /f́̈ıl/ est toujours motivé, est:

i) tout d'abord de reconnaître que ce type de ton flottant bas a la même valeur que le ton consonantique bas, et par conséquent, entraîne les mêmes effets de blocage du ton haut précédent: en d'autres termes, le ton flottant bas interne et les consonnes opaques exercent les mêmes types d'influence sur le ton haut précédent. Pourtant, comme nous l'avons montré, il n'y a aucune relation directe entre ces tons flottants bas et les consonnes opaques.

ii) ensuite, de reconnaître que tout ton périphérique bas, qui a été re-assigné à une autre unité porteuse par la règle de mapping ou recouvrement, s'affaiblit de fait et devient, par conséquent, incapable de bloquer la propagation du ton haut qui précède. Cela explique pourquoi le ton flottant bas dans les mots comme /dé / 'prends' en (30) et comme /níd/ 'individu' en (28) ne peuvent plus empêcher la propagation du ton haut précédent. 


\section{Conclusion}

Cet article soulève un problème de linguistique générale: l'origine et l'utilité des tons flottants en dagara. Pour aborder les faits, nous avons articulé le plan autour de trois points centraux: d'abord fournir dans la rubrique "remarques préalables" les informations d'ordre général pour la lecture de l'article; ensuite prouver qu'il existe effectivement des tons flottants, et enfin montrer que tout ton flottant bas n'autorise pas la propagation du ton haut précédent.

A la suite de la rubrique 'remarques préalables', on a établi l'existence des tons flottants bas à partir de leur effet d'abaissement de ton haut dans la combinatoire. Ainsi, on a retenu, d'abord, que parmi les bases verbales, certaines ont des tons flottants bas capables d'abaisser le ton haut qui suit, tandis que d'autres bases, apparemment de même structure que les premières, n'en ont qu'un seul, généralement un ton haut. Pour les bases nominales, nous avons montré que certaines n'ont qu'un ton haut, et avons mis en lumière le fait que d'autres ont, en plus du ton haut, un deuxième ton, un ton flottant bas qui provoque le downstep au sein du constituant syntaxique. Au sujet de ces dernières bases, il a été nécessaire d'établir un clivage: il y a des constituants nominaux à downstep qui maintiennent toujours le ton flottant bas dans les bases, et d'autres qui, parce que le downstep est lexicalisé, perdent le ton flottant bas quand les bases sont isolées de leurs suffixes.

Dans cette section, nous avons procédé à une relecture de la règle du mapping (ou recouvrement tonal) de Leben [1976], et avons montré que c'est cette règle même qui génère les tons flottants en dagara. En effet, il s'est avéré que lorsqu'il y a un seul ton dans la base, la règle consiste à imposer ce ton de base à tous les morphèmes, au détriment de leur(s) ton(s) qui devient/deviennent alors flottant(s). Quand, par contre, il y a deux tons dans la base, l'application de la règle consiste à imposer le deuxième ton de la base à tous les autres morphèmes au détriment de leur(s) propre(s) ton(s) également maintenu(s) flottant(s).

Pour aborder le dernier point, on a opéré une distinction entre les types de tons flottants. Ainsi, les tons flottants bas de tous les suffixes ont en commun le fait d'avoir subi un mouvement tonal de replacement dans une position périphérique. Il en est de même pour les tons flottants bas dans les bases, à la différence que dans les bases nominales, on a observé ceci: les constituants sans downstep n'ont que des bases à ton haut simple, alors que ceux impliquant un downstep ont un ton flottant bas qui s'élimine du système dans le cas du downstep lexicalisé, ou qui se maintient dans le système dans le cas du downstep (cf. 17) examiné en (21). C'est ce type de ton flottant qui a été décrit comme ton flottant interne-le terme interne signifiant que le ton en question occupe une position géographique située entre le ton de base et celui du suffixe.

Ensuite, nous avons démontré que les tons flottants bas périphériques en X1 ne peuvent pas bloquer le ton haut précédent et l'empêcher d'étendre son domaine sur X2, contrairement aux tons flottants bas internes qui, eux, ont la capacité de bloquer la propagation du ton haut précédent. On a d'ailleurs pu observer que dans des bases comme /dé / 'prends' (15a), (30), et dans des constituants comme /níd/ 'individu', (18) et (28), le ton haut précédent se propageait sur X2. Cette 
situation a soulevé une autre question théorique en ce qui concerne le point (c) du principe de bonne formation qui stipule que les traits d'association ne doivent pas se croiser. Pour que ce principe s'applique au dagara, il fallait d'abord résoudre le problème du ton flottant bas-celui en position finale de /dé/ ou de /níd/_-et poser une règle, la règle "d'alimentation" (feeding rule) qui, en procédant à l'élimination de ce ton flottant bas, permet à la règle de bonne formation de s'appliquer.

Il fallait enfin résoudre la question du lien éventuel entre ces tons flottants bas qui peuvent bloquer la propagation du ton haut précédent et les consonnes opaques qui entraînent les mêmes effets tonals. C'est alors que nous avons émis deux hypothèses. La première hypothèse qui stipule que les tons flottants bas internes proviennent des consonnes opaques, en l'occurrence $/ \mathrm{b}, \mathrm{d}, \mathrm{g} /$, a été écartée. La raison est que le ton bas consonantique en dagara, tout comme dans bon nombre de langues africaines (nupe, gen, par exemple), n'est actif qu'en position initiale et non pas en position médiane ni finale, comme c'est le cas de $/ \mathrm{b}$, d, g/ dans les mots reconstruits en dagara (cf. 43). On a, par contre, retenu la deuxième hypothèse qui consiste à poser que les tons flottants internes sont différents des autres parce qu'ils sont toujours motivés comme tons de dérivatifs qui sont toujours attestés, mais fondus dans les bases. Le locuteur dagara continue d'admettre que dans certaines bases, ce type de ton flottant bas existe et joue un rôle important de blocage du ton haut précédent. Cette hypothèse est corroborée par l'ensemble des données de la langue. On peut, par exemple, sur la base du clivage singulier/pluriel se rendre compte que lorsque le ton flottant bas n'est plus motivé comme ton de dérivatif, il disparaît au pluriel et n'entraîne plus de downstep, tel qu'indiqué en (25). Par contre, lorsque le ton flottant bas est toujours motivé comme en (26), il engendre toujours un downstep, même au pluriel. 


\section{RÉFÉRENCES}

Bonvini, Emilio. 1988. Prédication et énonciation en kàsım. Paris: Editions du C.N.R.S. (Sciences du Langage 1).

Bole-Richard, Remy. 1983. Systématique phonologique et grammaticale d'un parler ewe: le gen-mina du sud Togo et sud Benin. Paris: L'Harmattan.

Clements, G. N. and Kevin C. Ford. 1979. "Kikuyu tone shift and synchronic consequences." Linguistic Inquiry 10:179-210.

Delplanque, Alain. 1983. Phonologie transformationnelle du dagara (langue voltaïque du Burkina Faso). Paris: SELAF.

Delplanque, Alain. 1986. "La langue dagara. Essai de Sémiologie linguistique." Thèse d'État, Université de Paris VII.

Delplanque Alain and Benoît Ouoba. 1979. "Les classes nominales en gulmancema." Afrique et Langage 11:5-27.

de Saussure, Ferdinand. 1978. Cours de linguistique générale. Paris: Payothèque.

Goldsmith, John A. 1976. Autosegmental Phonology. Bloomington: Indiana University Linguistics Club.

Hagège Claude. 1982. La structure des langues. Paris: Presse Universitaire de France.

Houis, Maurice. 1977. "Plan de description des langues négro-africaines." Afrique et Langage 7:5-65.

Houis, Maurice. 1980. "Proposition pour une typologie des langues négro-africaines." Afrique et Langage 13:5-68.

Houis, Maurice. 1981. "La relation de détermination en syntaxe et composé nominaux." Afrique et Langage 16:5-47.

Houis, Maurice. 1983. "Description des unités significatives comme préalable à la lexicologie.' Afrique et Langage 19:5-49.

Hyman, Larry M. 1973. "The role of consonant types in natural tonal assimilations". In L. Hyman (ed.), Consonant Types and Tone. Los Angeles: Southern California Occasional Papers in Linguistics. Pp. 151-179.

Hyman Larry M. and Russell G. Schuh. 1974. "Universals of Tone Rules: Evidence From West Africa." Linguistic Inquiry 1:81-115. 
Hyman Larry M. and M. Tadadjeu, 1976. "Floatting tone in Mbam-Nkam." In Larry M. Hyman (ed.), Studies in Bantu Tonology (Southern California Occasional Papers in Linguistics 3). Los Angeles: University of Southern California Depatment of Linguistics. Pp. 57-111.

Jouannet, Francis. 1981. "Analyse paradigmatique ou analyse syntagmatique des tons." In Gladys Guarisma (ed.), Tons et accents dans les langues africaines. Paris: SELAF. Pp. 51-55.

Kaboré, Raphaël. 1981. Essai d'analyse de la langue mùvré (parler de Wàogò): Ouagadougou. Thèse d'état, Paris VII.

Kenstowicz Michael, E. Nikiéma, and M. Ourso. 1988. “Tonal polarity in two Gur Language." Studies in the Linguistic Sciences 18: 77-104.

Kinda Jules. 1983. "Dynamisme des tons et intonation en mooré (langue de hautevolta)." Thèse de 3ème cycle, Sorbonne nouvelle (Paris III).

Kiparsky, Paul. 1982. "Lexical phonology and morphology.” In I. S. Yang (ed.), Linguistics in the Morning Calm. Seoul: Hanshin. Pp. 3-91.

Kiparsky, Paul. 1985. "Some consequences of lexical phonology." Phonology Yearbook 2:83-138.

Leben, William R. 1973. "Suprasegmental phonology." Doctoral dissertation, M.I.T.

Leben, William R. 1976. "The representation of tone.” In Victoria Fromkin (ed.), Tone: a Linguistics survey. London: Academic Press. Pp. 177-220.

Manessy, Gabriel. 1969. Les langues gurunsi. Essai d'application de la méthode comparative à un groupe de langues. Paris: SELAF.

Manessy, Gabriel. 1975. Les langues Oti-volta, classification généalogique d'un groupe de langues voltaïques. Paris: SELAF.

Manessy, Gabriel. 1985. "Construction sérielle dans les langues africaines et les langues créoles.” Bulletin de la société linguistique de Paris 80,1:333-362.

Nikiéma, Norbert. 1989. "Structures radicales et processus morphologique en moore." Cahier du CERLESHS 2: 309-457 (Université de Ouagadougou. B.F.).

Odden, David. 1995. “Tone: African Languages.” In John A. Goldsmith (ed.), The Handbook of Phonological Theory. London: Blackwell. Pp. 445-475. 
O'Grady, William and Michael Dobrovosky. 1992. Contempory Linguistic Analysis: An Introduction. Copp Clark, Pitmant Ltd.

Paradis, Carole. 1984. "Le comportement tonal des constructions associatives en wobé." Journal of African Languages and Linguistics 6:147-171.

Peterson, Thomas. 1971. "Mooré Structure: A Generative Analysis of the TonalSystem and Aspects of Syntax." Doctoral Dissertation, UCLA.

Pulleyblank, Douglas. 1986. Tone in Lexical phonology, Dordrecht: Reidel.

Rialland, Annie. 1988. "Système prosodiques africains ou fondement empiriques pour un modèle multilinéaire." Doctorat d'état, Université de Nice.

Somé, Penou-Achille. 1982. Systématique du signifiant en dagara: variété wúlé. Paris: L'Harmattan, Agence de Coopération Culturelle et Technique.

Somé, Penou-Achille. 1989a. La parente pauvre en phonologie : l'occlusive glottale dans les langues voltaïques. Cahier du CERLESHS 2:216-307. (Université de Ouagadougou. B. F.)

Somé, Penou-Achille. 1989b. "Tonologie des propositions en syndèse en dagarawúlé." Cahier du CERLESHS 2:193-215. (Université de Ouagadougou. B. F.)

Somé, Penou-Achille. To appear. "Une procédure de découverte: détection des tons dans les schèmes tonals en dagara." Journal of West African Languages XXV.1: 3-41.

Somé, Penou-Achille. 1996. "Dérivation des bases en dagara: pourquoi et quand un deuxième ton?" Linguistique Africaine 17:43-106.

Somé, Penou-Achille. 1998. "L'influence des consonnes sur les tons en dagara, langue voltique du Burkina Faso." Studies in African Linguistics 27:3-47.

Somé, Der Joachim. 1975. "Contribution à l'étude phonologique du dagara." Mémoire de maîtrise, Université de Nice.

Somé, Der Joachim. 1983. "Description de la langue dagara de Haute Volta." Thèse de 3ème cycle, Université de Paris V, I \& II.

Stewart, John. M. 1983. "Downstep and floating low tone in Adjoukrou." Journal of African Languages and Linguistics 5:57-78.

Tchagbale, Zachari. 1984. "Lénition consonantique et amalgame dans l'analyse phonologique: le cas du tem." Annales de l'université d'Abidjan série $H$, linguistique 12:89-112. 
Tchagbale, Zachari. 1989. Manuel de la phonologie du trait distinctif, cours et exercices. MS. Abidjan: Université d'Abidjan.

Tadadjeu, Maurice. 1974. "Floatting tones, shifting rules, and downstep in Dschang-Bamileke." Studies in African Linguistics, supplement 5:283-290.

Williams, Edvin S. 1976. "Underlying tone in Margi and Igbo.” Linguistic Inquiry 7: 463-484.

Modern Languages Department

[Received November 2000;

University of Lethbridge accepted September 2002]

4401 University Drive

Lethbridge, Alberta

T1K 3M4

penouachille@hotmail.com 